\title{
Surface-induced melting and freezing II. A semi-empirical Landau-type model
}

\author{
B. Pluis, D. Frenkel and J.F. van der Veen \\ FOM-Institute for Atomic and Molecular Physics, Kruislaan 407, $1098 \mathrm{SJ}$ Amsterdam, The Netherlands
}

Received 16 January 1990; accepted for publication 13 July 1990

\begin{abstract}
Thermodynamic criteria are derived for the occurrence of surface-induced melting and freezing in a semi-infinite system. Use is made of a mean-field theory of the Landau-type. The parameters which enter the Landau free energy functional are expressed in terms of known interfacial free energies. Expressions are obtained for the order parameter profile in the surface region and for the temperature-dependent thickness of the molten (frozen) surface layer. The results explain recent observations of surface melting (or nonmelting) at differently oriented crystal faces of $\mathrm{Pb}$ and account for the general absence of surface-induced freezing effects in liquids.
\end{abstract}

\section{Introduction}

The phenomenon of surface-induced melting can be regarded as a wetting transition of the solid by its own melt. At temperatures well below the triple point $T_{\mathrm{m}}$ a microscopically thin liquid-like layer appears at the solid-vapor interface. As the temperature $T$ approaches $T_{\mathrm{m}}$ the layer thickness increases continuously and, in the case of complete wetting, eventually diverges. Infinitesimally close to $T_{\mathrm{m}}$ the melt front has penetrated deeply into the crystal, resulting in coexistence between essentially bulk solid, liquid and vapor phases. Conversely, a surface-induced freezing transition may occur for a liquid. In that case, as the temperature of the liquid is lowered toward $T_{m}$, an ordered solid-like layer forms on the free liquid surface and increases in thickness as $T_{\mathrm{m}}$ is approached.

While surface melting has been observed on a variety of crystal faces [1] and is believed to be a commonly occurring phenomenon, the opposite effect of surface-induced freezing is rarely seen. This asymmetric behavior of the surface with respect to melting and freezing is, very likely, the reason why a solid in general cannot be superheated, while a liquid can easily be undercooled.
In this paper a thermodynamic argument is presented which explains why surface-induced melting should indeed occur much more frequently than surface-induced freezing. It is shown under which circumstances these phenomena are expected to occur. Explicit expressions are obtained for the temperature dependence of the surface order parameter and the thickness of the melted (frozen) layer. In our model the Landau free-energy functional for semi-infinite bulk is taken as a starting point. The bulk and surface free energies entering the functional are assumed to be known functions of some scalar order parameter $M$, for which we take the Fourier component of the density which corresponds to the smallest nonzero reciprocal lattice vector.

Applying Landau theory to a semi-infinite system, Lipowsky and Speth [2] have shown that a first-order phase transition which in the bulk is characterized by a discontinuous jump in the order parameter may at the surface exhibit a continuous change in the order parameter. Hence, the surface may induce critical phenomena at a first-order bulk transition. For example, in the case of melting the bulk order parameter $M_{\mathrm{B}}$ jumps abruptly from a finite value to zero at the triple point $T_{\mathrm{m}}$, whereas at the surface the same parameter may 
continuously go to zero as $T \rightarrow T_{\mathrm{m}}$. The vanishing of the order parameter is accompanied by a divergence of the disordered layer thickness. The divergence has been predicted to be of logarithmic, $\ln \left[T_{\mathrm{m}} /\left(T_{\mathrm{m}}-T\right)\right]$, or of power-law form, $\left(T_{\mathrm{m}}-\right.$ $T)^{-r}$, depending on the range of atomic interactions (short or long range, respectively) $[3,4]$. Recent observations of surface melting on crystals of $\mathrm{Pb}$ [5-9], Ar [10], $\mathrm{Ne} \mathrm{[11]} \mathrm{and} \mathrm{Al}$ [12] have confirmed these predictions.

However, a major obstacle in any comparison with experimental data is the global way in which the existing Landau-type theories are formulated. The various parameters entering the Landau freeenergy functional usually are not specified, rendering a quantitative calculation of order parameter profiles versus temperature impossible. In the present work these parameters are correlated with the specific solid-vapor (sv), liquid-vapor (lv) and solid-liquid (sl) interface free energies $\gamma_{s v}, \gamma_{\mathrm{lv}}$ and $\gamma_{\mathrm{sl}}$, which are known for the respective two-phase equilibria and can be extrapolated to the temperature regime of interest. For the correlation length within the liquid phase, which enters the order parameter profile, an estimate is made through application of the Hansen-Verlet melting rule. A clear-cut criterion for the occurrence of a surfaceinduced melting transition is derived, which solely depends on the relative magnitudes of $\gamma_{\mathrm{sv}}, \gamma_{\mathrm{lv}}$ and $\gamma_{\mathrm{s} 1}$. The results of the calculations are found to be in good agreement with the experimental data on surface melting.

This paper is organized as follows. Section 2 gives the fundamental equations of the Landau theory for a semi-infinite system governed by short-range atomic interactions. From these equations, expressions for the surface order parameter, the equilibrium order parameter profile and the melted (frozen) layer thickness are derived in section 3. The expressions for the corresponding equilibrium Landau free energies are given in section 4, where also the relationships are established between the interfacial free energies, $\gamma_{\mathrm{sv}}, \gamma_{\mathrm{lv}}$ and $\gamma_{\mathrm{sl}}$ on the one hand, and the parameters entering the expressions in sections 2 and 3 on the other hand. Section 5 summarizes the calculations in a phase diagram in which the surface-induced melting and freezing regimes are indicated. In section
6 we discuss which elements of the periodic system are expected to exhibit surface-initiated melting and freezing and compare the predictions of the Landau model with various experimental observations. The role of long-range atomic interactions in surface melting is briefly discussed as well. In appendix A a semi-empirical expression for the correlation length within the liquid phase is given. Its value is estimated and compared with experimentally determined values. Finally, the possible influence of thermally induced interface fluctuations on the melted layer thickness is assessed in appendix B.

\section{Landau theory}

Consider a three-dimensional semi-infinite system of particles with short-range atomic interactions. The system is described by a scalar order parameter $M=M(z)$ which varies with the coordinate $z$ perpendicular to the surface. The surface is at $z=0$. The Landau free energy per unit area for such a system is given by the following functional of the order parameter $[2,13]$ :

$F(M)=f_{1}\left(M_{\mathrm{S}}\right)+\int_{0}^{\infty}\left[f(M)+\frac{1}{2} J\left(\frac{\mathrm{d} M}{\mathrm{~d} z}\right)^{2}\right] \mathrm{d} z$

Here, the term $f_{1}\left(M_{\mathrm{S}}\right)$ is the free energy contribution from the surface, with $M_{\mathrm{S}} \equiv M(z=0)$ the order parameter at the surface. The function $f(M)$ represents the free energy per unit volume for homogeneous bulk material. The second term in the integral takes into account the energy increase due to gradients in the order parameter, with $J$ a constant. The functional dependence of the bulk term $f(M)$ on $M$ is chosen to have the form of two parabolas of equal curvature [14]:

$f(M)=\frac{1}{2} \alpha M^{2}+\Lambda, \quad M \leq M^{*}$,

and

$f(M)=\frac{1}{2} \alpha\left(M_{\mathrm{cr}}-M\right)^{2}, \quad M \geq M^{*}$, 
with $\alpha>0$ and $M^{*}$ the intersection point of the two parabolas, given by

$M^{*}=\frac{1}{2} M_{\mathrm{cr}}-\frac{\Lambda}{\alpha M_{\mathrm{cr}}}$.

Here, $\Lambda$ represents the difference in free energy per unit volume between the solid and liquid phase. For temperatures $T$ close to $T_{\mathrm{m}}, \Lambda$ is approximately given by $\Lambda=L_{m}\left(1-T / T_{m}\right)$, with $L_{m}$ the latent heat of melting per unit volume. The temperature dependence of $f(M)$, as given by eq. (2a), is depicted in fig. 1. For $T<T_{\mathrm{m}}, f(M)$ has its minimum at $M=M_{\text {cr }}>0$, with $M_{\text {cr }}$ being the order parameter of the bulk in its crystalline state. For $T>T_{\mathrm{m}}, f(M)$ is minimal for $M=0$, which corresponds to the bulk being liquid. At $T=T_{m}$, $f(M)$ has two minima and there is coexistence between the two bulk phases. This functional form of $f(M)$ correctly models the first-order character of the bulk transition; as $T$ approaches $T_{\mathrm{m}}$ from below, the order parameter jumps discontinuously from $M_{\text {cr }}$ to zero at $T=T_{\mathrm{m}}$.

The surface term $f_{1}\left(M_{\mathrm{S}}\right)$ in eq. (1) is modeled as [2]

$f_{1}\left(M_{\mathrm{S}}\right)=\frac{1}{2} \alpha_{1} M_{\mathrm{S}}^{2}+C$,

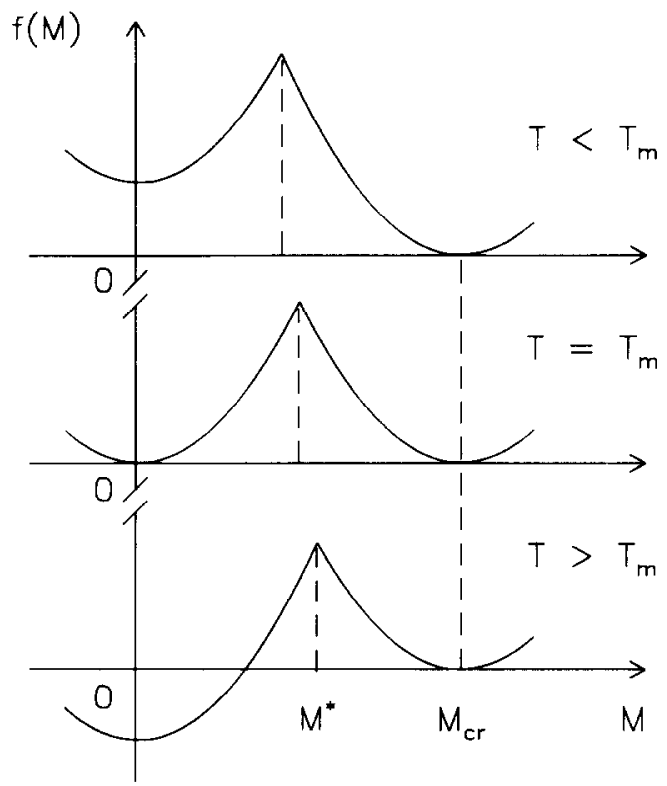

Fig. 1. The bulk term $f(M)$ in the Landau free energy for a first-order bulk transition at $T_{\mathrm{m}}$, for temperatures $T<T_{\mathrm{m}}$, $T=T_{\mathrm{m}}$ and $T>T_{\mathrm{m}}$

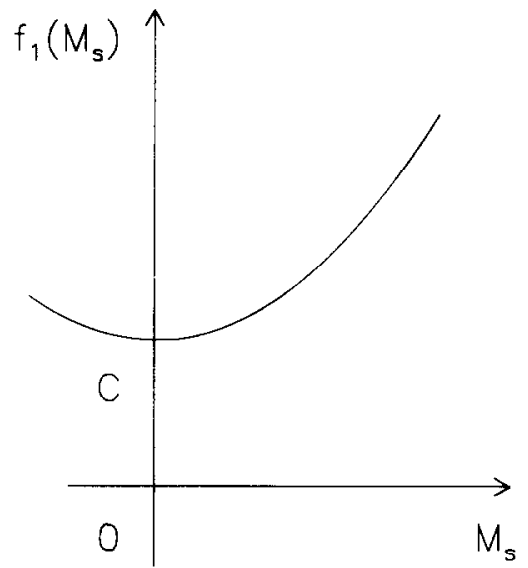

Fig. 2. The surface contribution $f_{1}\left(M_{\mathrm{S}}\right)$ to the Landau free energy functional.

where $\alpha_{1}$ and $C$ are constants which to a first approximation are assumed to be temperature-independent. For $\alpha_{1}>0$, eq. (4) describes the tendency of a surface to become disordered (see fig. 2), while for $\alpha_{1}<0$ the surface would like to be in an ordered state.

The equilibrium order parameter profile $M(z)$ is obtained by minimizing eq. (1) using the variational principle. This leads to the Euler equation [15]

$\frac{\partial f(M)}{\partial M}=J \frac{\mathrm{d}^{2} M}{\mathrm{~d} z^{2}}$

together with the boundary condition

$\frac{\partial f_{1}\left(M_{\mathrm{S}}\right)}{\partial M_{\mathrm{S}}}=\left.J \frac{\mathrm{d} M}{\mathrm{~d} z}\right|_{z=0}$.

Eq. (5) is analogous to the classical equation of motion of a particle with "coordinate" $M$ and "mass" $J$, moving in a "potential" $-f(M)$. The parameter $z$ represents "time". Conservation of "energy", which the additional requirement that $\left.(\mathrm{d} M / \mathrm{d} z)\right|_{z \rightarrow \infty}=0$, leads to [2]

$$
\frac{\mathrm{d} M}{\mathrm{~d} z}=\left\{\begin{array}{l}
+\left\{\left[2 f(M)-2 f\left(M_{\mathrm{B}}\right)\right] / J\right\}^{1 / 2}, \\
M_{\mathrm{S}}<M_{\mathrm{B}}, \\
-\left\{\left[2 f(M)-2 f\left(M_{\mathrm{B}}\right)\right] / J\right\}^{1 / 2}, \\
M_{\mathrm{S}}>M_{\mathrm{B}},
\end{array}\right.
$$


where $M_{\mathrm{B}} \equiv M(z \rightarrow \infty)$ is the order parameter in the bulk. The surface free energy $F_{\mathrm{s}}$ is obtained from the Landau free energy functional $F(M)$ by subtracting from the terms in the integral of eq. (1) the homogeneous bulk term $f\left(M_{\mathrm{B}}\right)$ and using eq. (7):

$F_{\mathrm{s}}=f_{1}\left(M_{\mathrm{S}}\right)+\int_{0}^{\infty} J\left(\frac{\mathrm{d} M}{\mathrm{~d} z}\right)^{2} \mathrm{~d} z$,

where $F_{\mathrm{s}}$ has its minimum value for the equilibrium order parameter profile.

\section{Order parameter profiles}

The order parameter profiles at equilibrium are obtained by integration of eq. (7), with the initial value $M_{\mathrm{S}}$ as determined by eq. (6). The profiles are most easily classified by the temperature dependence of the surface order parameter $M_{\mathrm{S}}$. Therefore, we first derive analytical expressions for the equilibrium values of $M_{\mathrm{S}}$ and then calculate and discuss the corresponding order parameter profiles $M(z)$.

\subsection{Surface melting}

The surface order parameter $M_{\mathrm{S}}$ at equilibrium is obtained by substituting eqs. (4) and (7) for $f_{1}\left(M_{\mathrm{S}}\right)$ and $\left.(\mathrm{d} M / \mathrm{d} z)\right|_{z=0}$ in the left- and righthand side of eq. (6), respectively, and then solving for $M_{\mathrm{S}}$. For $T<T_{\mathrm{m}}(\Lambda>0)$ and $0 \leq M_{\mathrm{S}} \leq M^{*}$ one finds

$M_{\mathrm{S}}=\left(\frac{2 J \Lambda}{\alpha_{1}^{2}-J \alpha}\right)^{1 / 2}$,

on condition that

$\alpha_{1}>(J \alpha)^{1 / 2}$ and

$\Lambda \leq \Lambda_{\max } \equiv \frac{\alpha M_{\mathrm{cr}}^{2}\left[\alpha_{1}-(J \alpha)^{1 / 2}\right]}{2\left[\alpha_{1}+(J \alpha)^{1 / 2}\right]}$.

Note that the surface order parameter $M_{\mathrm{S}}$ depends on the temperature through the parameter $\Lambda=$ $L_{\mathrm{m}}\left(1-T / T_{\mathrm{m}}\right)$. As $T \rightarrow T_{\mathrm{m}}\left(\Lambda \rightarrow 0^{+}\right), M_{\mathrm{S}}$ decreases continuously to zero. By contrast, the bulk order parameter $M_{\mathrm{B}}$ jumps abruptly from $M_{\mathrm{cr}}$ to

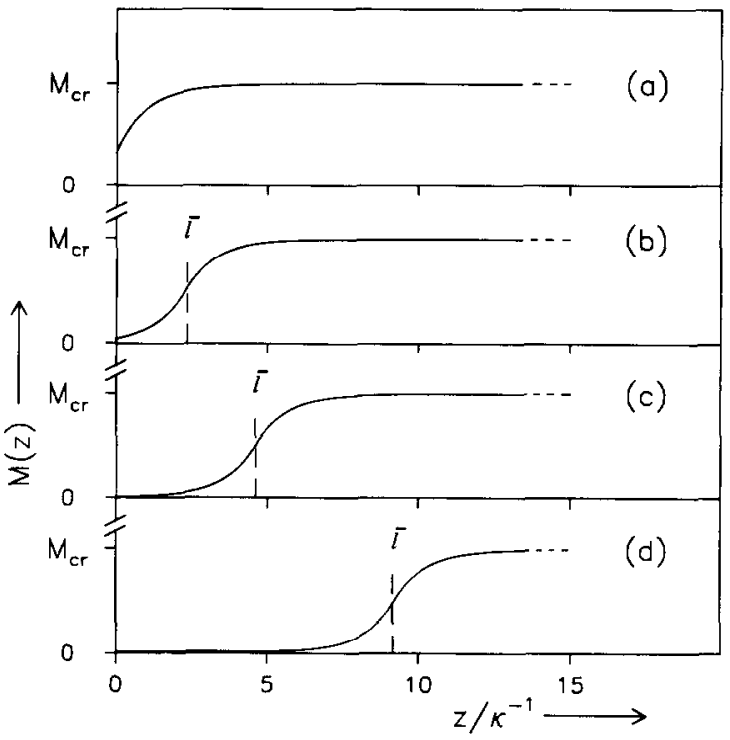

Fig. 3. Order parameter profiles $M(z)$ in reduced units of $\kappa^{-1}$ for a surface melting transition. The profiles are evaluated from eq. (11) for $\alpha_{1} /(J \alpha)^{1 / 2}=2.0$ and (a) $\Lambda / \Lambda_{\max }=1.0$, (b) $\Lambda / \Lambda_{\max }=10^{-2}$, (c) $\Lambda / \Lambda_{\max }=10^{-4}$, and (d) $\Lambda / \Lambda_{\max }=10^{-8}$. The diverging position of the solid-quasi-liquid interface is indicated by $\bar{l}$.

zero at the transition temperature. 'The equilibrium order parameter profile is found by integration of cq. (7), with $M_{\mathrm{S}}$ in eq. (9) as the boundary value:

$$
\begin{aligned}
& M(z)=\frac{1}{2} M_{\mathrm{cr}} \mathrm{e}^{\kappa(z-l)}-\frac{\Lambda}{\alpha M_{\mathrm{cr}}} \mathrm{e}^{-\kappa(z-l)}, \quad z \leq \bar{l}, \\
& M(z)=M_{\mathrm{cr}}-\left(\frac{1}{2} M_{\mathrm{cr}}+\frac{\Lambda}{\alpha M_{\mathrm{cr}}}\right) \mathrm{e}^{-\kappa(z-l)}, z \geq \bar{l},
\end{aligned}
$$

with $\kappa \equiv(\alpha / J)^{1 / 2}$. Here, $\bar{l}$ is defined as the depth at which $M(z=\bar{l})=M^{*}$. It is the depth at which the order parameter reaches about half of its value $M_{\text {cr }}$ in the bulk. As such, $\bar{l}$ is a good measure of the equilibrium thickness of the disordered layer. It is given by

$i=\frac{1}{2} \kappa^{1} \ln \left(\Lambda_{\max } / \Lambda\right)$.

The temperature dependence of the order parameter profile is shown in figs. $3 a-3 d$. The profiles were evaluated from eq. (11) with use of eq. (10) by taking a constant value for $\alpha_{1} /$ 
$(J \alpha)^{1 / 2}=2.0$, but different values for $\Lambda$, namely $\Lambda / \Lambda_{\max }=10^{\circ}, 10^{-2}, 10^{-4}$ and $10^{-8}$. As $\Lambda$ decreases, i.e. $T$ increases, the surface order parameter decreases continuously from $M^{*}$ at $\Lambda=\Lambda_{\max }$ (fig. 3a) to zero as $\Lambda \rightarrow 0^{+}$, at which point $T$ approaches $T_{\mathrm{m}}$ (fig. $3 \mathrm{~d}$ ). At all temperatures, except $T_{\mathrm{m}}$ itself, the surface order parameter has a nonzero value. Hence, the surface region should be regarded as a quasi-liquid with properties intermediate between those of the solid and the liquid. The smooth decrease of the surface order parameter is accompanied by a continuous increase of the quasi-liquid layer thickens $\dot{l}$. As the temperature increases the quasi-liquid layer thickness continuously from the surface inwards (see fig. 3). The layer thickness diverges logarithmically as the temperature approaches the melting point $T_{\mathrm{m}}$ (eq. (12)). This transition is therefore classified as a surface-induced melting transition. It can be regarded as a special case of complete wetting [16].

An important parameter is $\kappa^{-1}$, which is the distance over which $M(z)$ in the disordered phase changes by a factor $e$ (see eq. (11a)). We identify $\kappa^{-1}=(J / \alpha)^{1 / 2}$ as the correlation length $\xi_{\ell}$ within the liquid phase.

\subsection{Surface nonmelting}

For temperatures $T<T_{\mathrm{m}}$ and $M_{\mathrm{S}} \geq M^{*}$ the order parameter at the surface is given by

$M_{\mathrm{S}}=\frac{(J \alpha)^{1 / 2}}{\alpha_{1}+(J \alpha)^{1 / 2}} M_{\mathrm{cr}}$,

on condition that

$-(J \alpha)^{1 / 2}<\alpha_{1} \leq(J \alpha)^{1 / 2}$ and $\Lambda>0$,

or

$\alpha_{1} \geq(J \alpha)^{1 / 2}$ and

$\Lambda \geq \Lambda_{\max } \equiv \frac{\alpha M_{\mathrm{cr}}^{2}\left[\alpha_{1}-(J \alpha)^{1 / 2}\right]}{2\left[\alpha_{1}+(J \alpha)^{1 / 2}\right]}$.

According to eq. (13) $M_{\mathrm{S}}$ does not depend on the temperature.

The order parameter profile is given by

$M(z)=M_{\text {cr }}-M_{\text {cr }} \frac{\alpha_{1}}{(J \alpha)^{1 / 2}+\alpha_{1}} \mathrm{e}^{-\kappa z}$.

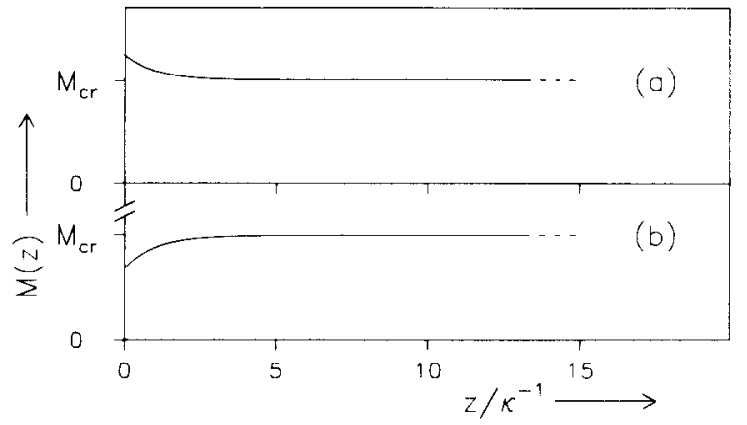

Fig. 4. Order parameter profiles $M(z)$ in reduced units of $\kappa^{-1}$ for a surface nonmelting transition. The profiles are obtained from eq. (15) for (a) $\alpha_{1} /(J \alpha)^{1 / 2}=-0.1$ and (b) $\alpha_{1} /(J \alpha)^{1 / 2}$ $=0.5$.

The profile is shown in figs. $4 \mathrm{a}$ and $4 \mathrm{~b}$ for $\alpha_{1} /$ $(J \alpha)^{1 / 2}=-0.1$ and $\alpha_{1} /(J \alpha)^{1 / 2}=0.5$, respectively. The order parameter decreases or increases monotonically from the value $M_{\mathrm{S}}$ at $z=0$, to the value $M_{\mathrm{cr}}$ in the bulk. The surface region is solidlike. Furthermore, the profile is independent of temperature and the surface exhibits no critical melting behavior. Hence, we call this transition a surface nonmelting transition. It represents a case of nonwetting [16].

\subsection{Surface freezing}

For temperatures $T>T_{\mathrm{m}}(\Lambda<0)$ the bulk is liquid. Consider $M_{\mathrm{S}} \geq M^{*}$, then $M_{\mathrm{S}}$ has two solutions

$M_{\mathrm{S}}^{ \pm}=\frac{-J \alpha M_{\mathrm{cr}} \pm\left[\alpha_{1}^{2} J \alpha M_{\mathrm{cr}}^{2}-2 J \Lambda\left(\alpha_{1}^{2}-J \alpha\right)\right]^{1 / 2}}{\alpha_{1}^{2}-J \alpha}$,

under the restrictive conditions that

$-(J \alpha)^{1 / 2}<\alpha_{1}<0$ and $\Lambda_{\text {min }} \leq \Lambda<0$,

with

$\Lambda_{\min } \equiv \frac{\alpha \alpha_{1}^{2} M_{\mathrm{cr}}^{2}}{2\left(\alpha_{1}^{2}-J \alpha\right)}$.

For small values of $|\Lambda|$ the solutions of eq. (16) 
for the order parameter $M_{\mathrm{S}}$ at the surface are to a first approximation given by

$M_{\mathrm{S}}^{-}=\frac{(J \alpha)^{1 / 2}}{(J \alpha)^{1 / 2}-\alpha_{1}} M_{\mathrm{cr}}+\frac{J \Lambda}{(J \alpha)^{1 / 2} \alpha_{1} M_{\mathrm{cr}}}+\mathrm{O}\left(\Lambda^{2}\right)$

and

$M_{\mathrm{S}}^{+}=\frac{(J \alpha)^{1 / 2}}{(J \alpha)^{1 / 2}+\alpha_{1}} M_{\mathrm{cr}}-\frac{J \Lambda}{(J \alpha)^{1 / 2} \alpha_{1} M_{\mathrm{cr}}}+\mathrm{O}\left(\Lambda^{2}\right)$

It will be shown in section 4 that $M_{\mathrm{S}}^{+}$is the relevant solution since it corresponds to the state of lowest surface free energy.

The order parameter profile, with $M_{\mathrm{S}}=M_{\mathrm{S}}^{+}$as boundary value (valid in the limit of small $|\Lambda|$ ), is given by

$$
\begin{aligned}
M^{+}(z)= & M_{\text {cr }}+\left(\frac{-\Lambda}{2 \alpha}\right)^{1 / 2} \mathrm{e}^{-\kappa\left(z-z^{\prime}\right)} \\
& -\left(\frac{-\Lambda}{2 \alpha}\right)^{1 / 2} \mathrm{e}^{\kappa\left(z-z^{\prime}\right)} \\
& -\frac{\Lambda}{\alpha M_{\text {cr }}}\left(\frac{(J \alpha)^{1 / 2}-\alpha_{1}}{2 \alpha_{1}} \mathrm{e}^{-\kappa z}\right)
\end{aligned}
$$

$0 \leq z \leq z^{\prime}$,

$$
\begin{aligned}
M^{+}(z)= & M_{\mathrm{cr}}+\left(\frac{-\Lambda}{2 \alpha}\right)^{1 / 2} \mathrm{e}^{-\kappa\left(z-z^{\prime}\right)} \\
& -\left(\frac{-\Lambda}{2 \alpha}\right)^{1 / 2} \mathrm{e}^{\kappa\left(z-z^{\prime}\right)}
\end{aligned}
$$

$z^{\prime} \leq z \leq \tilde{s}$

$M^{+}(z)=\left(\frac{1}{2} M_{\text {cr }}-\frac{\Lambda}{\alpha M_{\text {cr }}}\right) \mathrm{e}^{-\kappa(z-\bar{s})}$,

$z \geq \bar{s}$,

with

$z^{\prime}=\kappa^{-1} \ln \left[\frac{-\alpha_{1} M_{\mathrm{cr}}}{\left[\alpha_{1}+(J \alpha)^{1 / 2}\right]}\left(\frac{2 \alpha}{-\Lambda}\right)^{1 / 2}\right]$,

being the depth for which $M^{+}\left(z=z^{\prime}\right)=M_{\mathrm{cr}}$ and $\bar{s}=z^{\prime}+\kappa^{-1} \ln \left[M_{\mathrm{cr}}\left(\frac{\alpha}{-2 \Lambda}\right)^{1 / 2}\right]$,

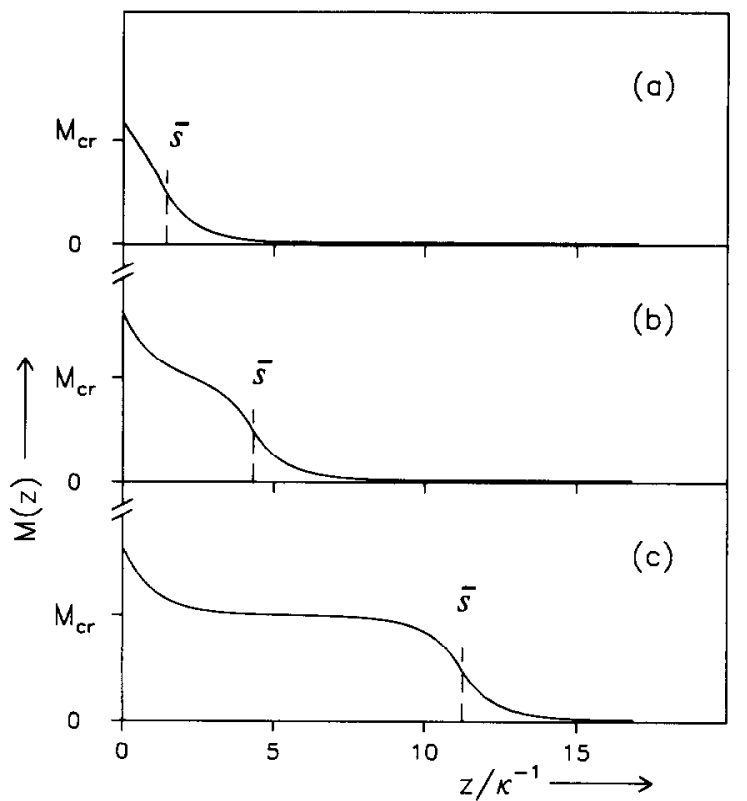

Fig. 5. Order parameter profiles $M(z)$ in reduced units of $\kappa^{-1}$ for a surface freezing transition. The profiles, given by eq. (20), are evaluated with $\alpha_{1} /(J \alpha)^{1 / 2}$ fixed to the value of -0.4 and for different temperatures (a) $\Lambda / \Lambda_{\min }=1.0$, (b) $\Lambda / \Lambda_{\min }=$ $10^{-1}$, and (c) $\Lambda / \Lambda_{\min }=10^{-4}$. The position of the quasisolid-liquid interface, indicated by $\bar{s}$, diverges for $\Lambda / \Lambda_{\min } \rightarrow 0$.

being the depth at which $M^{+}(z=\bar{s})=M^{*}$. Here, $\bar{s}$ represents the equilibrium thickness of the quasi-solid layer. Note that the order parameter profile has a discontinuity at $z=z^{\prime}$ which is proportional to $|\Lambda| \mathrm{e}^{-\kappa z^{\prime}}-\mathrm{O}\left(|\Lambda|^{3 / 2}\right)$ (see eqs. (20a) and (20b)). This is caused by the fact that in the derivation of eq. (20a) the approximation for $M_{\mathrm{S}}$ $=M_{\mathrm{S}}^{+}$is used which only includes the leading order term in $|\Lambda|$ (eq. (19)).

The temperature dependence of the order parameter profile $\mathrm{M}^{+}(z)$ is shown in figs. 5a-5c. The order parameter profiles were calculated from eq. (20) and with use of eq. (17) by taking a constant value for $\alpha_{1} /(J \alpha)^{1 / 2}=-0.4$, but different values for $\Lambda$, namely $\Lambda / \Lambda_{\text {min }}=10^{\circ}, 10^{-1}$, and $10^{-4}$. As the temperature decreases the surface order parameter increases continuously from $M_{\mathrm{S}}$ $=J \alpha M_{\mathrm{cr}} /\left(J \alpha-\alpha_{1}^{2}\right)$ at $\Lambda=\Lambda_{\min }$ (see eq. (16) and fig. 5a), to $M_{\mathrm{S}}^{+}=(J \alpha)^{1 / 2} M_{\mathrm{cr}} /\left[(J \alpha)^{1 / 2}+\alpha_{1}\right]$ in the limit $\Lambda \rightarrow 0^{-}$(see eq. (19) and fig. 5c). Simultaneously, the position of the quasi-solid-liquid interface progresses continuously from the surface 
inwards and a frozen layer is formed at the liquid surface. The thickness $\bar{s}$ of this layer increases logarithmically with decreasing temperature and diverges as $T_{\mathrm{m}}$ is approached. This transition is therefore classified as surface-induced freezing. It can be regarded as a special case of complete drying [17]. The parameter $\kappa^{-1}=(J / \alpha)^{1 / 2}$ entering eqs. (20b) and (22) is the length over which $M(z)$ decays from a value of $M_{\text {cr }}$ in the surface region to a value $1 / e$ smaller at greater depth. Hence, we identify $\kappa^{-1}$ as the correlation length $\xi_{\text {s }}$ within the solid phase. (We note that the equality $\xi_{\ell}=\xi_{\mathrm{s}}$ arises from our choice of two parabolas of equal curvature $\alpha$ for the bulk free energy $f(M)$, see eqs. (2a) and (2b) and fig. 1. If we were to have taken unequal curvatures $\alpha_{\ell}$ and $\alpha_{\mathrm{s}}$ for the parabolas centered around $M=0$ and $M=M_{\mathrm{cr}}$, respectively, then we would have obtained $\xi_{\ell}=$ $\left(J / \alpha_{\ell}\right)^{1 / 2}$ and $\xi_{\mathrm{s}}=\left(J / \alpha_{\mathrm{s}}\right)^{1 / 2}$, and the expressions for the order parameter profiles would be slightly different.)

\subsection{Surface nonfreezing}

If $T>T_{\mathrm{m}}(\Lambda<0)$ and $M_{\mathrm{S}} \leq M^{*}$ then the order parameter $M_{\mathrm{S}}$ at the surface must be zero, provided $\alpha_{1} \neq-(J \alpha)^{1 / 2}$. The corresponding order parameter profile is given by

$M(z)=M_{\mathrm{S}} \mathrm{e}^{-\kappa z}=0$

and is independent of temperature. Consequently, the surface region remains liquid and there are no critical surface effects as the temperature approaches $T_{\mathrm{m}}$. We classify this transition therefore as a surface nonfreezing transition. It represents a special case of nondrying [17].

\section{Surface free energy}

The surface free energy is evaluated for the various surface transitions by inserting the corresponding equilibrium order parameter profiles, as found in section 3 , in eq. (8) and performing the integration over depth $z$. Explicit relations are derived between the parameters $\alpha_{1},(J \alpha)^{1 / 2}$ and $M_{\mathrm{cr}}$ on the one side, and the semi-experimentally known interfacial free energies per unit area, $\gamma_{\mathrm{sv}}$, $\gamma_{\mathrm{sl}}$ and $\gamma_{\mathrm{lv}}$, on the other side. In addition, expressions for the surface free energy and the equilibrium thickness are given as functions of the latter parameters.

\subsection{Surface melting and nonmelting}

The minimum surface free energy for the case of surface-initiated melting is obtained by inserting in eq. (8) the equilibrium order parameter profile as given by eq. (11):

$$
\begin{aligned}
F_{\mathrm{s}}= & C+\frac{1}{4} M_{\mathrm{cr}}^{2}(J \alpha)^{1 / 2}+\bar{l} \Lambda \\
& +\frac{1}{4} M_{\mathrm{cr}}^{2}(J \alpha)^{1 / 2} \frac{\alpha_{1}-(J \alpha)^{1 / 2}}{\alpha_{1}+(J \alpha)^{1 / 2}} \mathrm{e}^{-2 \kappa l},
\end{aligned}
$$

with $\bar{l}$ the equilibrium thickness of the quasi-liquid layer (eq. (12)). $\Lambda$ s the temperature $T$ approaches $T_{\mathrm{m}}, \Lambda \rightarrow 0^{+}$and the equilibrium thickness diverges logarithmically as $\bar{l} \sim|\ln \Lambda|$. In this limit the third and fourth terms of eq. (24) vanish. Hence, we find

$$
F_{\mathrm{s}}=C+\frac{1}{4} M_{\mathrm{cr}}^{2}(J \alpha)^{1 / 2} \text { for } T \rightarrow T_{\mathrm{m}} .
$$

The corresponding equilibrium order parameter profile, with $M_{\mathrm{S}}=0$, is that of an infinitely thick liquid layer in contact with the solid. There are two interfaces, namely the liquid-vapor interface at the surface and the solid-liquid interface in the bulk. The equilibrium free energy at $T=T_{\mathrm{m}}$ can therefore be expressed as

$F_{\mathrm{s}}=\gamma_{\mathrm{lv}}+\gamma_{\mathrm{sl}}$ for $T \rightarrow T_{\mathrm{m}}$,

with $\gamma_{1 v}$ and $\gamma_{s 1}$ the free energies of the liquidvapor and solid-liquid interface, respectively. Comparing expressions (25) and (26) and considering the fact that the parameter $C$ in eq. (25) represents the surface term $f_{1}\left(M_{\mathrm{S}}=0\right)$ in the Landau free energy (eq. (4)), one obtains

$C=\gamma_{\mathrm{lv}}$

and

$\frac{1}{4} M_{\mathrm{cr}}^{2}(J \alpha)^{1 / 2}=\gamma_{\mathrm{sl}}$.

At $T=T_{\mathrm{m}}$ the interface free energies $\gamma_{\mathrm{lv}}$ and $\gamma_{\mathrm{sl}}$ refer to the respective two-phase equilibria and can be determined experimentally (see section 7.1) [18-22]. 
In the case of surface nonmelting, with $T<T_{\mathrm{m}}$ and the order parameter profile $M(z)$ given by eq. (15), the equilibrium surface free energy is given by

$F_{\mathrm{s}}=C+\frac{1}{2} M_{\mathrm{cr}}^{2}(J \alpha)^{1 / 2} \frac{\alpha_{1}}{(J \alpha)^{1 / 2}+\alpha_{1}}$,

independent of temperature. The corresponding order parameter profile represents an essentially solid-like surface region (see fig. 4). Therefore, the equilibrium free energy can be equated to the solid-vapor interface free energy $\gamma_{\mathrm{sv}}$ :

$C+\frac{1}{2} M_{\mathrm{cr}}^{2}(J \alpha)^{1 / 2} \frac{\alpha_{1}}{(J \alpha)^{1 / 2}+\alpha_{1}}=\gamma_{\mathrm{sv}}$.

Combination of eqs. (27), (28) and (30) yields the following useful relations:

$\frac{\alpha_{1}}{\alpha_{1}+(J \alpha)^{1 / 2}}=\frac{\gamma_{s v}-\gamma_{1 v}}{2 \gamma_{s l}}$

and

$\frac{\alpha_{1}-(J \alpha)^{1 / 2}}{\alpha_{1}+(J \alpha)^{1 / 2}}=\frac{\gamma_{s v}-\gamma_{s l}-\gamma_{1 v}}{\gamma_{s l}}$.

Consider again the case of surface melting. We can now rewrite the surface free energy, as given by eq. (24), in terms of interfacial free energies:

$F_{\mathrm{s}}=\gamma_{\mathrm{sl}}+\gamma_{\mathrm{lv}}+L_{\mathrm{m}}\left(1-T / T_{\mathrm{m}}\right) \bar{l}+\Delta \gamma \mathrm{e}^{-2 \kappa \bar{l}}$,

where we have introduced again $\Lambda=L_{\mathrm{m}}(1-T /$ $T_{m}$ ), with $L_{m}$ the latent heat of melting per unit volume. The parameter $\Delta \gamma=\gamma_{\mathrm{sv}}-\gamma_{\mathrm{sl}}-\gamma_{\mathrm{lv}}$ is the interface free energy which the ordered solid surface has in excess of a surface completely wetted by an infinitely thick liquid layer. It is important to note that the interfacial free energies $\gamma_{\mathrm{sv}}, \gamma_{\mathrm{sl}}$ and $\gamma_{\mathrm{lv}}$ in eqs. (31) and (32) are not equilibrium quantities. At temperatures where the surface is wetted by a quasi-liquid layer of finite thickness the solid-vapor, solid-liquid and liquid-vapor interfaces to which the $\gamma$ values refer are unstable and do, in fact, not exist. However, $\gamma_{s v}$ can be estimated by extrapolating the equilibrium $\gamma_{\mathrm{sv}}$ value which is known at lower temperatures, to the temperature regime of interest [23]. Likewise, the $\gamma_{\mathrm{lv}}$ and $\gamma_{\mathrm{sl}}$ values which are known at $T=T_{\mathrm{m}}$ can be extrapolated to $T<T_{\mathrm{m}}$, but, since surface

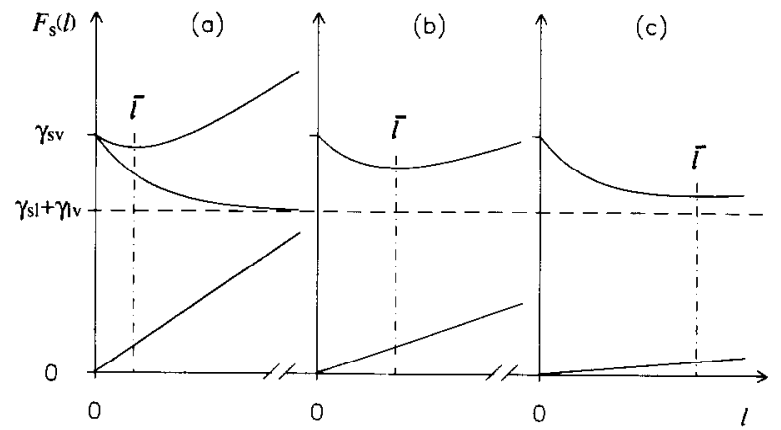

Fig. 6. The surface free energy $F_{s}(l)$ (eq. (34)) as a function of the thickness $l$ of the quasi-liquid layer (not to scale). The straight solid line is the undercooling contribution to $F_{\mathrm{s}}(l)$. The exponentially decaying curve represents the sum of $\gamma_{\mathrm{sl}}+\gamma_{\mathrm{lv}}$ (dashed line) and $\Delta \gamma \mathrm{e}^{-2 \kappa l}$. The equilibrium thickness of the quasi-liquid layer $l=i$ for which $F_{\mathrm{s}}(l)$ has its minimum increases with rising temperature (panels (a) to (c)).

melting occurs relatively close to $T_{\mathrm{m}}$, the values at $T_{\mathrm{m}}$ can be taken [21,22].

The equilibrium thickness $l$ can also be expressed in terms of the above interfacial free energies. Substituting eqs. (28) and (31b) in eq. (12) we obtain

$\bar{l}=\frac{\xi_{\ell}}{2} \ln \left(\frac{2 \Delta \gamma T_{\mathrm{m}}}{L_{\mathrm{m}}\left(T_{\mathrm{m}}-T\right) \xi_{\ell}}\right)$,

with $\xi_{\ell}=\kappa^{-1}$ the correlation length within the liquid phase (see section 3.1 and appendix A). It is readily verified that the surface free energy is minimal for the layer thickness given by eq. (33). Let $F_{\mathrm{s}}(l)$ be the free energy per unit area for a system with arbitrary quasi-liquid layer thickness $l$ :

$F_{\mathrm{s}}(l)=\gamma_{\mathrm{sl}}+\gamma_{\mathrm{lv}}+L_{\mathrm{m}}\left(1-T / T_{\mathrm{m}}\right) l+\Delta \gamma \mathrm{e}^{-2 \kappa l}$.

Then $\mathrm{d} F_{\mathrm{s}}(l) / \mathrm{d} l=0$ for $l=\bar{l}$. The different energy terms in eq. (34) are sketched in fig. 6 as a function of $l$. The third term represents the free energy cost associated with undercooling the liquid and increases linearly with $l$. It leads to an effective attractive force per unit area between the solid-quasi-liquid and quasi-liquid-vapor interfaces which is equal to $-L_{m}\left(1-T / T_{m}\right)$. The fourth term represents the gain in interfacial energy associated with the dry solid surface being wetted by a quasi-liquid. This term leads to an 
exponentially decaying force of magnitude $2 \kappa \Delta \gamma \mathrm{e}^{-2 \kappa l}$ between the two interfaces. For $\Delta \gamma>0$ this force is repulsive, driving the interfaces apart. The equilibrium thickness $\dot{l}$ is the thickness for which the net force $\mathrm{d} F_{\mathrm{s}}(l) / \mathrm{d} l$ is zero. As the temperature $T$ approaches $T_{\mathrm{m}}$ more closely, $i$ increases continuously as shown in figs. $6 \mathrm{~b}$ and $6 \mathrm{c}$.

If $\Delta \gamma \leq 0$ then, according to eq. (32) and the above argument, it will only cost energy to have a quasi-liquid layer on the surface. Hence, the surface is non melting under these conditions. Note that our earlier criteria $\alpha_{1}>(J \alpha)^{1 / 2}$ and $-(J \alpha)^{1 / 2}$ $<\alpha_{1} \leq(J \alpha)^{1 / 2}$ for melting and nonmelting (eqs. (10) and (14a)) are equivalent to the conditions $\Delta \gamma>0$ and $\Delta \gamma \leq 0$, respectively (see eq. (31b)).

\subsection{Surface freezing and nonfreezing}

For the case that $-(J \alpha)^{1 / 2}<\alpha_{1}<0$ and $\Lambda_{\text {min }}$ $\leq \Lambda<0$ there are three possible solutions for the surface order parameter, namely $M_{\mathrm{S}}^{-}, M_{\mathrm{S}}^{+}$(eqs. (18), (19)) and $M_{\mathrm{S}}=0$, with the three corresponding order parameter profiles $M^{-}(z), M^{+}(z)$ and $M(z)=0$. The profiles $M^{-}(z)$ and $M^{+}(z)$ describe a surface freezing transition, whereas the profile $M(z)=0$ corresponds to a surface nonfreezing transition. Substitution of these profiles into the general expression for the surface free energy (eq. (8)) yields for the profile $M^{+}(z)$ the lowest surface free energy, provided $\alpha_{1}$ and $\Lambda$ are in the range

$-(J \alpha)^{1 / 2}<\alpha_{1}<-\frac{1}{3}(J \alpha)^{1 / 2}$

and

$\Lambda_{\min } \leq \Lambda<0$,

with $\Lambda_{\min }$ as defined in eq. (17). Under these conditions $M^{+}(z)$ is the equilibrium profile. For $\alpha_{1}$ and $\Lambda$ values outside this range the flat profile $M(z)=0$ is energetically the most stable one. The order parameter profile $M^{-}(z)$ can therefore be discarded.

For the case of surface freezing the equilibrium surface free energy is obtained by substituting the order parameter profile $M^{+}(z)$ into eq. (8):

$$
\begin{aligned}
F_{\mathrm{s}}= & C+\frac{1}{4} M_{\mathrm{cr}}^{2}(J \alpha)^{1 / 2}+\frac{1}{2} M_{\mathrm{cr}}^{2}(J \alpha)^{1 / 2} \frac{\alpha_{1}}{(J \alpha)^{1 / 2}+\alpha_{1}} \\
& -\Lambda \bar{s}-M_{\mathrm{cr}}^{2}(J \alpha)^{1 / 2} \frac{\alpha_{1}}{\alpha_{1}+(J \alpha)^{1 / 2}} \mathrm{e}^{-\kappa \bar{s}}, \quad(36)
\end{aligned}
$$

with $\bar{s}$ being the equilibrium thickness of the quasi-solid laycr. As for the case of surface melting the surface free energy can be rewritten in terms of interfacial free energies. Substituting eqs. (27), (28) and (30) into eq. (36), we obtain

$$
\begin{aligned}
F_{\mathrm{s}}= & \gamma_{\mathrm{sl}}+\gamma_{\mathrm{sv}}-L_{\mathrm{m}}\left(1-T / T_{\mathrm{m}}\right) \bar{s} \\
& +2\left(\gamma_{\mathrm{lv}}-\gamma_{\mathrm{sv}}\right) \mathrm{e}^{-\kappa \bar{s}} .
\end{aligned}
$$

As $T$ approaches $T_{\mathrm{m}}\left(\Lambda \rightarrow 0^{-}\right)$the layer thickness $\bar{s}$ diverges logarithmically (eqs. (21) and (22)) and the last two terms in eq. (37) vanish. In this limit the free energy of the system is simply given by $F_{\mathrm{s}}=\gamma_{\mathrm{sl}}+\gamma_{\mathrm{sv}}$; the surface is then covered by an infinitely thick crystalline film and the system consists of two interfaces, namely the solid-vapor interface at the surface and solid-liquid interface in the bulk.

The equilibrium thickness of the quasi-solid layer, as given by eqs. (21) and (22), can also be expressed in terms of interfacial free energies. By substitution of eqs. (27), (28) and (30) into eqs. (21) and (22) one obtains

$\bar{s}=\xi_{\mathrm{s}} \ln \left(\frac{2\left(\gamma_{\mathrm{lv}}-\gamma_{\mathrm{sv}}\right) T_{\mathrm{m}}}{L_{\mathrm{m}}\left(T-T_{\mathrm{m}}\right) \xi_{\mathrm{s}}}\right)$,

with $\xi_{\mathrm{s}}=\kappa^{-1}$ representing the correlation length within the solid phase. It is readily checked that $\bar{s}$ is the thickness giving minimal surface free energy. Let $F_{\mathrm{s}}(s)$ be the surface free energy per unit area for a system with arbitrary quasi-solid layer thickness $s$ :

$$
\begin{aligned}
F_{\mathrm{s}}(s)= & \gamma_{\mathrm{sl}}+\gamma_{\mathrm{sv}}-L_{\mathrm{m}}\left(1-T / T_{\mathrm{m}}\right) s \\
& +2\left(\gamma_{\mathrm{lv}}-\gamma_{\mathrm{sv}}\right) \mathrm{e}^{-\kappa s},
\end{aligned}
$$

then minimizing $F_{\mathrm{s}}(s)$ with respect to $s$ yields $s=\bar{s}$. The third term in eq. (39) is associated with superheating the solid above its melting point. This contribution favors small values of $s$. The fourth term represents the change in interfacial free energy associated with the liquid surface becoming quasi-solid. In the case of surface freezing $\alpha_{1}<-1 / 3(J \alpha)^{1 / 2}$ (see eq. (35)). Substitution of this condition into eq. (31) yields the surface freezing criterion $\Delta \gamma^{\prime} \equiv \gamma_{1 \mathrm{v}}-\gamma_{\mathrm{sl}}-\gamma_{\mathrm{sv}}>0$. In that case $\gamma_{\mathrm{lv}}>\gamma_{\mathrm{sv}}$ and the fourth term in eq. (39) favors large values of $s$ because the system gains interfacial free energy if the liquid substrate is covered by a quasi-solid film. 


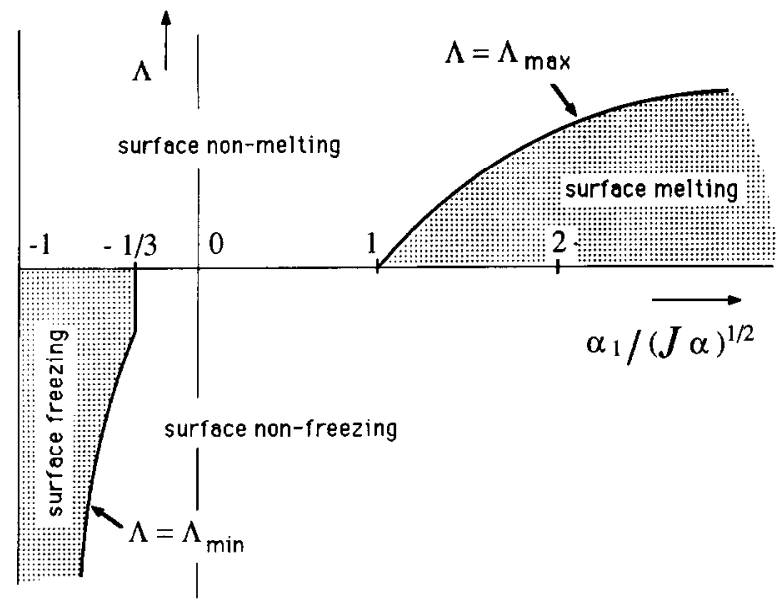

Fig. 7. Phase diagram in which the different types of surface phase transitions are indicated. The regions in which surface melting and surface freezing occur are shaded. For definition of symbols, see text.

For the case of surface nonfreezing $M(z)$ is zero for all $z$. Substituting this profile into eq. (8) we find, as expected, for the equilibrium surface free energy

$F_{\mathrm{s}}=C=\gamma_{1 \mathrm{v}}$

Note that for the case of nonfreezing $\alpha_{1} \geq$ $-1 / 3(J \alpha)^{1 / 2}$, i.e., $\Delta \gamma^{\prime}=\gamma_{\mathrm{lv}}-\gamma_{\mathrm{sl}}-\gamma_{\mathrm{sv}} \leq 0$ (see eq. (31)). Thus it costs energy to have a solidified layer on top of the liquid substrate.

\section{Phase diagram}

To summarize the results we present a phase diagram in which the different types of phase transitions are indicated, together with their respective conditions of appearance. In fig. 7 we have plotted along the vertical and horizontal axes the parameters $\Lambda$ and $\alpha_{1} /(J \alpha)^{1 / 2}$, respectively. The undercooling energy $\Lambda=L_{m}\left(1-T / T_{m}\right)$ is a linear function of the temperature, while $\alpha_{1} /$ $(J \alpha)^{1 / 2}$ is assumed to be temperature-independent.

First consider the upper half of the phase diagram in which $\Lambda>0\left(T<T_{\mathrm{m}}\right)$. There are two surface transitions, surface melting and surface non melting, which occur in two different regions separated by the curve $\Lambda=\Lambda_{\max } . \Lambda_{\max }$ becomes zero for $\alpha_{1} /(J \alpha)^{1 / 2}=1$, which corresponds to the condition $\Delta \gamma=0$ (see eq. (31)). As $T$ approaches $T_{\mathrm{m}}$ from below the sign of $\Delta \gamma$ is of crucial importance since it determines whether there is a surface melting transition or not. For, if $\Delta \gamma>0\left(\alpha_{1} /\right.$ $\left.(J \alpha)^{1 / 2}>1\right)$, the surface region starts to disorder when $\Lambda$ becomes smaller than $\Lambda_{\max }$. That is, the disordering sets in as soon as the temperature becomes larger than the onset temperature $T_{0}$, with $T_{0}$ defined by $\Lambda_{\max }=L_{m}\left(1-T_{0} / T_{m}\right)$ and given by

$T_{0}=T_{\mathrm{m}}-\frac{2 \Delta \gamma T_{\mathrm{m}}}{L_{\mathrm{m}} \xi_{\ell}}$

Eq. (41) is readily derived from eqs. (10), (28) and (31). Clearly, for $\Delta \gamma>0, T_{0}$ is smaller than $T_{\mathrm{m}}$ and a surface melting transition appears in the temperature range $T_{0}<T<T_{\mathrm{m}}$. On the other hand, if $\Delta \gamma \leq 0\left(\alpha_{1} /(J \alpha)^{1 / 2} \leq 1\right)$ the surface remains well-ordered up to $T_{\mathrm{m}}$. Therefore, a clear-cut criterion for the occurrence of surface melting or nonmelting is given by the sign of the excess free energy $\Delta \gamma$; surface melting will occur only for $\Delta \gamma>0$.

Secondly, consider the lower half of the phase diagram in which $\Lambda<0$ (or $T>T_{\mathrm{m}}$ ). For $\alpha_{1} /$ $(J \alpha)^{1 / 2}<-1 / 3$ there are two phase transitions, namely surface freezing and surface nonfreezing which occur in the regions separated by the curve $\Lambda=\Lambda_{\text {min }}$. The condition $\alpha_{1} /(J \alpha)^{1 / 2}<-1 / 3$ (eq. (35)) corresponds to $\Delta \gamma^{\prime}=\gamma_{\mathrm{lv}}-\gamma_{\mathrm{sl}}-\gamma_{\mathrm{sv}}>0$, with $\Delta \gamma^{\prime}$ representing the free energy which the liquid surface has in excess of a surface covered by an infinitely thick solid film. By analogy to the case of surface melting, $\Delta \gamma^{\prime}$ is the parameter that determines whether or not there is a surface freezing transition, as $T_{\mathrm{m}}$ is approached. If $\Delta \gamma^{\prime} \leq 0$, the surface remains liquid, whereas for $\Delta \gamma^{\prime}>0$ a frozen layer is formed at the surface.

Finally, we note that in the phase region $\alpha_{1} /$ $(J \alpha)^{1 / 2} \leq-1$ the Landau expressions yield unphysical results. The presence of this unphysical regime in the phase diagram is a consequence of our simple choice for the form of the surface term $f_{1}\left(M_{\mathrm{s}}\right)$ which enters the Landau free-energy functional of eq. (1). 
Table 1

Table of specific interfacial free energies $\gamma_{\mathrm{sv}}, \gamma_{\mathrm{sl}}$ and $\gamma_{\mathrm{lv}}$ at $T_{\mathrm{m}}$ for a number of common elements [21-23] (the sign of the excess free energies $\Delta \gamma \equiv \gamma_{\mathrm{sv}}-\gamma_{\mathrm{sl}}-\gamma_{\mathrm{lv}}$ and $\Delta \gamma^{\prime} \equiv \gamma_{\mathrm{Iv}}-\gamma_{\mathrm{sl}}-\gamma_{\mathrm{sv}}$ determines whether or not there are surface melting and surface freezing transitions, respectively)

\begin{tabular}{|c|c|c|c|c|c|c|c|c|}
\hline $\bar{Z}$ & & $\begin{array}{l}\gamma_{\mathrm{sv}} \\
\left(\mathrm{mJ} / \mathrm{m}^{2}\right)\end{array}$ & $\begin{array}{l}\gamma_{\mathrm{sl}} \\
\left(\mathrm{mJ} / \mathrm{m}^{2}\right)\end{array}$ & $\begin{array}{l}\gamma_{\mathrm{lv}} \\
\left(\mathrm{mJ} / \mathrm{m}^{2}\right)\end{array}$ & $\begin{array}{l}\Delta \gamma \\
\left(\mathrm{mJ} / \mathrm{m}^{2}\right)\end{array}$ & $\begin{array}{l}\Delta \gamma \\
\left(\mathrm{mJ} / \mathrm{m}^{2}\right)\end{array}$ & $\begin{array}{l}\text { Melting }(+) \\
\text { Nonmelting }(-)\end{array}$ & $\begin{array}{l}\text { Freezing }(+) \\
\text { Nonfreezing }(-)\end{array}$ \\
\hline 11 & $\mathrm{Na}$ & 223 & 31 & 200 & -8 & -54 & $(-)$ & $(-)$ \\
\hline 12 & $\mathrm{Mg}$ & 679 & 115 & 570 & -6 & -224 & $(-)$ & $(-)$ \\
\hline 13 & $\mathrm{Al}$ & 1032 & 154 & 865 & 13 & -321 & $(+)$ & $(-)$ \\
\hline 14 & $\mathrm{Si}$ & 1038 & 416 & 800 & -178 & -654 & $(-)$ & $(-)$ \\
\hline 23 & V & 2280 & 375 & 1900 & 5 & -703 & $(+)$ & $(-)$ \\
\hline 24 & $\mathrm{Cr}$ & 2031 & 381 & 1700 & -50 & -712 & $(-)$ & $(-)$ \\
\hline 25 & $\mathrm{Mn}$ & 1297 & 183 & 1100 & 14 & -380 & $(+)$ & $(-)$ \\
\hline 26 & $\mathrm{Fe}$ & 2206 & 326 & 1830 & 50 & -702 & $(+)$ & $(-)$ \\
\hline 27 & $\mathrm{Co}$ & 2197 & 345 & 1830 & 22 & -712 & $(+)$ & $(-)$ \\
\hline 28 & $\mathrm{Ni}$ & 2104 & 356 & 1750 & -2 & -710 & $(-)$ & $(-)$ \\
\hline 29 & $\mathrm{Cu}$ & 1592 & 263 & 1310 & 19 & -545 & $(+)$ & $(-)$ \\
\hline 30 & $\mathrm{Zn}$ & 895 & 119 & 770 & 6 & -244 & $(+)$ & $(-)$ \\
\hline 31 & $\mathrm{Ga}$ & 794 & 58 & 715 & 21 & -137 & $(+)$ & $(-)$ \\
\hline 32 & $\mathrm{Ge}$ & 870 & 273 & 640 & -43 & -503 & $(-)$ & $(-)$ \\
\hline 41 & $\mathrm{Nb}$ & 2314 & 399 & 1960 & -45 & -753 & $(-)$ & $(-)$ \\
\hline 42 & Mo & 2546 & 490 & 2130 & -74 & -816 & $(-)$ & $(-)$ \\
\hline 44 & $\mathrm{Ru}$ & 2591 & 443 & 2250 & -102 & -784 & $(-)$ & $(-)$ \\
\hline 45 & $\mathbf{R h}$ & 2392 & 384 & 1970 & 38 & -806 & $(+)$ & $(-)$ \\
\hline 46 & $\mathrm{Pd}$ & 1808 & 302 & 1480 & 26 & -630 & $(+)$ & $(-)$ \\
\hline 47 & $\mathrm{Ag}$ & 1065 & 184 & 910 & -29 & -339 & $(-)$ & $(-)$ \\
\hline 48 & $\mathrm{Cd}$ & 697 & 81 & 590 & 26 & -188 & $(+)$ & $(-)$ \\
\hline 49 & In & 638 & 48 & 560 & 30 & -126 & $(+)$ & $(-)$ \\
\hline 50 & $\mathrm{Sn}$ & 654 & 66 & 570 & 18 & -150 & $(+)$ & $(-)$ \\
\hline 73 & $\mathrm{Ta}$ & 2595 & 477 & 2180 & -62 & -892 & $(-)$ & $(-)$ \\
\hline 74 & W & 2753 & 590 & 2340 & -178 & -1003 & $(-)$ & $(-)$ \\
\hline 75 & $\operatorname{Re}$ & 3100 & 591 & 2650 & -141 & -1041 & $(-)$ & $(-)$ \\
\hline 76 & Os & 3055 & 566 & 2500 & -12 & -1121 & $(-)$ & $(-)$ \\
\hline 77 & $\mathrm{Ir}$ & 2664 & 466 & 2250 & -52 & -880 & $(-)$ & $(-)$ \\
\hline 78 & $\mathrm{Pt}$ & 2223 & 334 & 1860 & 29 & -674 & $(+)$ & $(-)$ \\
\hline 79 & $\mathrm{Au}$ & 1363 & 200 & 1130 & 33 & -433 & $(+)$ & $(-)$ \\
\hline 81 & $\mathrm{Tl}$ & 547 & 66 & 465 & 16 & -148 & $(+)$ & $(-)$ \\
\hline 82 & $\mathrm{~Pb}$ & 544 & 62 & 460 & 22 & -146 & $(+)$ & $(-)$ \\
\hline 83 & $\mathrm{Bi}$ & 501 & 74 & 380 & 47 & -195 & $(+)$ & $(-)$ \\
\hline
\end{tabular}

\section{Discussion}

\subsection{Which elements exhibit surface-initiated melting or freezing?}

In order to predict which elements of the periodic system show surface melting or surface freezing one has to evaluate the criteria for which these two phenomena are expected to occur. The sign of $\Delta \gamma\left(\Delta \gamma^{\prime}\right)$ determines whether or not surface-ini- tiated melting (freezing) occurs. Prediction of the sign of $\Delta \gamma$ and $\Delta \gamma^{\prime}$ requires knowledge of the interface free energies $\gamma_{\mathrm{sv}}, \gamma_{\mathrm{sl}}$ and $\gamma_{\mathrm{lv}}$. The liquid-vapor interface free energy $\gamma_{\mathrm{lv}}$ is known for most elements through measurements of the surface tension [22,24]. The solid-liquid interface free energy $\gamma_{\mathrm{sl}}$ is much less accurately known. Several methods have been used to determine $\gamma_{\mathrm{s} 1}$ $[18,19]$. The one most frequently employed is measuring the amount of undercooling before homo- 
geneous nucleation of the solid occurs $[18,21]$. The least accessible quantity is the solid-vapor interface free energy $\gamma_{\mathrm{sv}}$. Semi-empirical estimates, for $T=0$ and averaged over different surface orientations, have been given by Miedema for a large number of elements, along with a prescription to calculate their temperature dependence [23]. Using these estimates we extrapolate the value for $\gamma_{\mathrm{sv}}$ at $T=0$ to its value near $T_{\mathrm{m}}$ [23].

Estimates of $\gamma_{\mathrm{sv}}, \gamma_{\mathrm{sl}}$ and $\gamma_{\mathrm{lv}}$, based on Miedema's data [21-23], are listed in table 1 for several elements, together with the signs of $\Delta \gamma$ and $\Delta \gamma^{\prime}$. There is a large uncertainty in the sign of $\Delta \gamma$ if its value is close to zero. This is because $\Delta \gamma$ is the difference between nearly equal values of $\gamma_{\mathrm{sv}}$ and $\gamma_{\mathrm{sl}}+\gamma_{\mathrm{lv}}$ and especially the former one is not accurately known. In addition, crystal faces with open packing of atoms will have higher $\gamma_{\mathrm{sv}}$ and $\gamma_{\mathrm{sl}}$ values than close-packed faces, see section 6.2. Hence, the table should only be used as a crude indicator for the melting (+) or nonmelting (-) of a crystal face with average packing of atoms. On the other hand, the sign predicted for $\Delta \gamma^{\prime}$ is much more reliable. Of the elements listed in table 1 several are expected to exhibit a surface melting transition, but none shows a surface freezing transition. Experiments confirm the predictions of table 1. Surface-induced melting has been observed on crystals of $\mathrm{Pb}[5-9,25-28], \mathrm{Cu}[29], \mathrm{Al}[12,30]$, and $\mathrm{Ga}$ [31], while numerous undercooling experiments on a variety of elements indicate a general absence of surface freezing effects in liquids $[18,20]$.

\subsection{Crystal-face and temperature dependence of surface-initiated melting}

The excess free energy $\Delta \gamma$ for a given element will vary with the orientation $\{h k l\}$ of the crystal face:

$\Delta \gamma^{\{h k l\}}=\gamma_{\mathrm{sv}}^{\{h k l\}}-\gamma_{\mathrm{s}}^{\{h k l\}}-\gamma_{\mathrm{Iv}}$,

where both $\gamma_{\mathrm{sv}}^{\{h k l\}}$ and $\gamma_{\mathrm{s}}^{\{h k l\}}$ are anisotropic. The variation in $\Delta \gamma^{\{h k l\}}$ with orientation is mainly determined by the anisotropy in $\gamma_{\mathrm{sv}}^{[h k l]}$ because $\gamma_{s \mathrm{v}}^{\{h k l\}} \gg \gamma_{s \mid}^{\{h k l\}}[21,23]$. For metals the anisotropy of $\gamma_{\mathrm{sv}}^{\{h k l\}}$ is only a few percent at maximum, with the most open crystal face always having the highest $\gamma_{\mathrm{sv}}^{\{h k l\}}$ value [32-36]. Yet, a small anisotropy in $\gamma_{s v}^{\{h k l\}}$ can lead to a large percentage variation in $\Delta \gamma^{\{h k l\}}$ and thus to a strong variation in melting behavior with surface orientation. For example, in the case of $\mathrm{Pb}$ the maximum anisotropy of $\gamma_{\mathrm{sv}}^{\{h k l\}}$ measured amounts to 6\% [32]. This corresponds to a variation of $\sim 33 \mathrm{~mJ} / \mathrm{m}^{2}$ in $\gamma_{\mathrm{sv}}^{\{k k l\}}$ (see table 1), which is comparable to the tabulated value of $22 \mathrm{~mJ} / \mathrm{m}^{2}$ for $\Delta \gamma$ itself. Therefore, a small anisotropy in $\gamma_{\mathrm{sv}}^{\{h k l\}}$ may even cause a reversal in the sign of $\Delta \gamma$, resulting in melting and nonmelting of the differently oriented surfaces on one and the same crystal. According to table 1 there are many elements for which $|\Delta \gamma|$ is small. For these elements, the open-packed crystal faces having the largest $\gamma_{\mathrm{sv}}^{\{h k l\}}$ are expected to melt readily, while the close-packed faces with the smallest $\gamma_{\mathrm{sv}}^{\{h k l\}}$ may not melt at all below $T_{\mathrm{m}}$.

The melted layer thickness, as given by eq. (33), depends on the crystal face orientation through the anisotropic excess free energy $\Delta \gamma^{\{h k l\}}$. This dependence has been seen in medium-energy ion scattering investigations of the melting of differently oriented crystal faces of $\mathrm{Pb}$, sec part $\mathrm{I}$ of this investigation. For temperatures close to $T_{\mathrm{m}}$ the open-packed faces show a pronounced surface melting transition with large quasi-liquid layer thickness, while the crystal faces with orientations close to the densely packed $\{111\}$ and $\{100\}$ planes are nonmelting. Other metals such as Al [12] and $\mathrm{Cu}$ [29] show a very similar orientation dependence of the melting behavior of the surface.

The quasi-liquid layer thickness should increase logarithmically with temperature and diverge at $T=T_{\mathrm{m}}$. The logarithmic increase, as predicted by eq. (33), has indeed been observed for $\mathrm{Pb}$ [5-7] and $\mathrm{Al}$ [12]. One of the parameters controlling the rate at which the quasi-liquid thickness grows logarithmically is the correlation length $\xi_{\ell}$ within the liquid phase. For $\mathrm{Pb}\{110\}$ the value of $\xi_{\ell}$ has experimentally been determined to be $0.63 \mathrm{~nm}$. This value is consistent with the estimated value of $\xi_{\ell}=0.9 \pm 0.4 \mathrm{~nm}$ which is obtained through application of the Hansen-Verlet melting rule (see appendix $\mathrm{A}$ ).

Recently, a cross-over from a logarithmic to a power-law growth was observed on $\mathrm{Pb}\{110\}$ [6]. 
The power law stems from long-range dispersion forces and will be discussed in section 6.4 .

\subsection{Order parameter profile for surface-induced melting}

The order parameter profile for surface melting is given by eq. (11). By substituting eq. (28) into eq. (11) and using the relations $\Lambda=L_{m}\left(1-T / T_{m}\right)$ and $\xi_{\ell}=\kappa^{-1}=(J / \alpha)^{1 / 2}$ we obtain the following expression for the profile:

$$
\begin{aligned}
M(z)= & \frac{1}{2} M_{\mathrm{cr}} \mathrm{e}^{(z-\bar{l}) / \xi_{\ell}} \\
& -\frac{1}{2} M_{\mathrm{cr}}\left(\frac{L_{\mathrm{m}}\left(T_{\mathrm{m}}-T\right) \xi_{\ell}}{2 T_{\mathrm{m}} \gamma_{\mathrm{sl}}}\right) \mathrm{e}^{-(z-i) / \xi_{\ell}}, \\
z \leq i, & \\
M(z)= & M_{\mathrm{cr}}-\frac{1}{2} M_{\mathrm{cr}} \mathrm{e}^{-(z-i) / \xi_{\ell}} \\
& -\frac{1}{2} M_{\mathrm{cr}}\left(\frac{L_{\mathrm{m}}\left(T_{\mathrm{m}}-T\right) \xi_{\ell}}{2 T_{\mathrm{m}} \gamma_{\mathrm{sl}}}\right) \mathrm{e}^{-(z-i) / \xi_{\ell}},
\end{aligned}
$$

$z \geq \bar{l}$

which $\bar{l}$ given by eq. (33). The order parameter profile can be calculated as a function of temperature if $L_{\mathrm{m}}, \xi_{\ell}, \gamma_{\mathrm{s}}^{\{h k l\}}$ and $\Delta \gamma^{\{h k l\}}$ are known. For example, in the case of $\mathrm{Pb}\{110\}$ we take $L_{\mathrm{m}}=2.47$ $\times 10^{8} \mathrm{~J} / \mathrm{m}^{3}[24], \quad \gamma_{s}\{110\} \approx 60 \times 10^{-3} \mathrm{~J} / \mathrm{m}^{2}$ [20], $\xi_{\ell}=0.63 \mathrm{~nm}$ and $\Delta \gamma^{\{110\}}=21.2 \times 10^{-3} \mathrm{~J} / \mathrm{m}^{2}[6]$. For these values the last term in eq. (43a) and (43b) can be neglected in the temperature regime $T_{\mathrm{m}}-T \leq 20 \mathrm{~K}$. Therefore, close to $T_{\mathrm{m}}$ the profile solely depends on $\xi_{\ell}$ and $\bar{l}$. Fig. 8 shows the calculated order parameter profile, normalized to $M_{\text {cr }}$, for $T=600.4 \mathrm{~K}\left(T_{\mathrm{m}}=600.7 \mathrm{~K}\right)$. At this temperature the quasi-liquid film thickness equals $\bar{l}=$ $2.0 \mathrm{~nm}$ (see eq. (33)). The width of the order parameter profile, as marked by the $16 \%$ and $84 \%$ levels of the normalized order parameter, equals $-1.4 \mathrm{~nm}$. This value is consistent with the experimental value of $\sim 1.75 \mathrm{~nm}$ ( $\sim 10$ monolayers), as determined from a detailed shape analysis of measured energy spectra of backscattered protons from $\mathrm{Pb}\{110\}$ close to $T_{\mathrm{m}}$ [9]. The $16 \%-84 \%$ width of the order parameter profile, however, is larger than the upper limit for the $16 \%-84 \%$ width of the solid-quasi-liquid profile of the average density

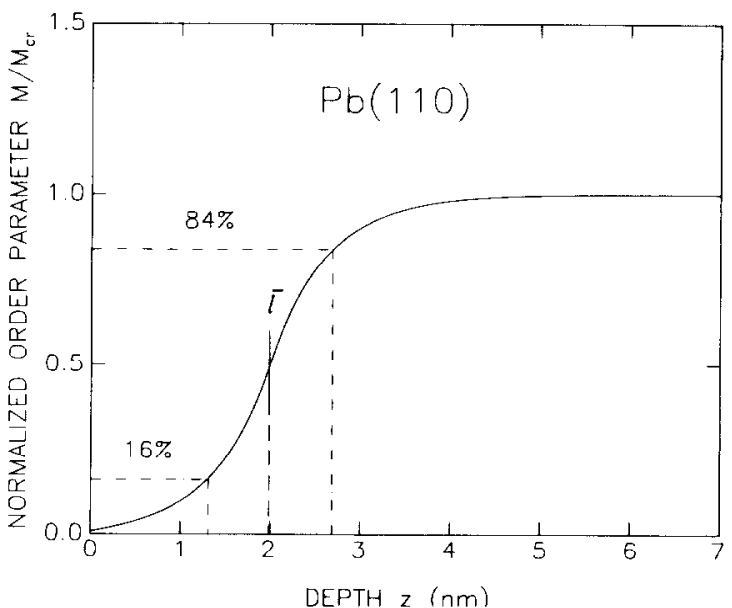

Fig. 8. The crystallinity profile for $\mathrm{Pb}\{110\}$, normalized to the bulk crystallinity $M_{\mathrm{cr}}$. The profile, calculated using eq. (43), is given for $\xi_{\ell}=0.63 \mathrm{~nm}, \Delta \gamma^{\{110\}}=21.2 \times 10^{-3} \mathrm{~J} / \mathrm{m}^{2}[6]$ and $T=T_{\mathrm{m}}-0.3 \mathrm{~K}$. The quasi-liquid layer thickness is $\bar{l}=2 \mathrm{~nm}$ (eq. (33)). The horizontal dash-dotted lines indicate the $16 \%$ and $84 \%$ levels of the normalized order parameter. The corresponding $16 \%-84 \%$ width of the solid-quasi-liquid interface equals $\sim 1.4 \mathrm{~nm}$.

$(<0.9 \mathrm{~nm})$, as obtained from a recent X-ray reflectivity study [37].

\subsection{Role of long-range atomic interactions in surface-induced melting}

In the foregoing we discussed only the effect of short-range atomic interactions on the melting behavior. However, in each system containing particles, whether it is a metal, insulator or semi-conductor, there are also long-range atomic interactions, namely induced-dipole-induced-dipole or van der Waals interactions [4]. The long-range van der Waals interactions are attractive and fall off as $-\epsilon(\lambda / r)^{6}$, with $\epsilon$ and $\lambda$ the energy and length scale of the pair potential and $r$ the interparticle separation. The effect of the long-range interactions on the shape of the order parameter profiles can be neglected for $|\bar{l}-z| \leq \xi_{\ell}$, because near to the quasi-liquid-solid interface the short-range interactions dominate. At large distances from the interface, i.e., for $|\bar{l}-z| \gg \xi_{\ell}$, the van der Waals interactions become important and cause the tails in the order parameter profile to change from an exponential form $\mathrm{e}^{-\kappa z}$ to a power-law form $z^{-3}$ [38-40]. 
The long-range atomic interactions, in the case of surface-induced melting, lead to an additional contribution to the surface free energy $F_{\mathrm{s}}(l)$ (eq. (34)). In the limit of large quasi-liquid layer thickness $l$ the surface free energy becomes

$$
\begin{aligned}
F_{\mathrm{s}}(l)= & \gamma_{\mathrm{sl}}+\gamma_{\mathrm{lv}}+L_{\mathrm{m}}\left(1-T / T_{\mathrm{m}}\right) l+\Delta \gamma^{\mathrm{SR}} \mathrm{e}^{-2 \kappa l} \\
& +\Delta \gamma^{\mathrm{LR}} A l^{-2}
\end{aligned}
$$

where $A$ is a positive constant and $\Delta \gamma^{\mathrm{SR}}$ and $\Delta \gamma^{\mathrm{LR}}$ are the contributions from the short-range (SR) and long-range (LR) atomic interactions to the excess free energy $\Delta \gamma \equiv \Delta \gamma^{\mathrm{T}}$, with $\Delta \gamma^{\mathrm{T}}=\Delta \gamma^{\mathrm{SR}}$ $+\Delta \gamma^{\text {LR }}$. The last term in eq. (44) represents the additional contribution to $F_{\mathrm{s}}(l)$ due to long-range atomic interactions [41], with $\Delta \gamma^{\mathrm{LR}} A=W$, which is assumed to be independent of the orientation $\{h k l\}$ of the surface. The parameter $W$ is the Hamaker constant and is approximately given by $[3,41]$

$$
W=\frac{\pi}{12} \rho_{\ell}\left(\rho_{\mathrm{s}}-\rho_{\ell}\right) \epsilon \lambda^{6}
$$

with $\rho_{\mathrm{s}}$ and $\rho_{\ell}$ the average particle number densities in the solid and liquid phase, respectively. Eq. (45) is readily obtained by using a simple stepfunction model for the density profile [41].

In the derivation of the long-range contribution to $F_{\mathrm{s}}(l)$ (eq. (44)) nonretarded interactions were assumed. However, retardation effects become important if the quasi-liquid layer thickness becomes sufficiently large (typically $l \geq 5 \mathrm{~nm}$ ) [4]. In that case the time taken by the electric field to cross the layer becomes comparable to the period of the fluctuating dipole itself. This makes the induceddipole-induced-dipole interactions less attractive. As a result, the $l^{-2}$ dependence of the last term of eq. (44) changes into a $l^{-3}$ dependence, as $l$ increases further.

The condition for surface-induced melting is given by $\Delta \gamma^{\mathrm{SR}}>0$. Then the short-range contribution to $F_{\mathrm{s}}(l)$ leads to an effective force between the solid-quasi-liquid and quasi-liquid-vapor interfaces which is repulsive in that it favors large values of $l$ (see section 4.1). For $\rho_{\mathrm{s}}>\rho_{\ell}, W>0$ and the long-range contribution leads to a repulsive force as well. Minimizing $F_{\mathrm{s}}(l)$ with respect to $l$ yields the equilibrium thickness $l=\bar{l}$. Let us define the cross-over thickness $l_{\mathrm{c}}$ as the thickness for which the long-range contribution to $\mathrm{d} F_{\mathrm{s}}(l) / \mathrm{d} l$ is equal to the short-range contribution. For larger thicknesses $l>l_{\mathrm{c}}$ the short-range contribution becomes negligible and the equilibrium thickness is then given by

$\bar{l}=\left(\frac{\left(T_{\mathrm{m}}-T\right) L_{\mathrm{m}}}{2 T_{\mathrm{m}} W}\right)^{-1 / 3}$.

A cross-over from a logarithmic growth to powerlaw growth has indeed heen observed for $\mathrm{Pb}\{110\}$ [6], biphenyl [42], and for thin $\mathrm{Ne}$ films [11]. Retardation effects in the fluctuating dipole interactions ultimately cause the exponent of the power law to change from $-1 / 3$ to $-1 / 4$, as $T_{\mathrm{m}}$ is approached [4].

So far we only discussed the case $\Delta \gamma^{\mathrm{SR}}>0$ and $\mathrm{W}>0$, i.e., $\Delta \gamma^{\mathrm{T}}=\Delta \gamma^{\mathrm{SR}}+\Delta \gamma^{\mathrm{LR}}>0$. In principle there are three other possibilities, namely (a) $\Delta \gamma^{\text {SR }}$ $>0$ and $W<0$, (b) $\Delta \gamma^{\text {SR }}<0$ and $W>0$ and (c) $\Delta \gamma^{\mathrm{SR}}<0$ and $W<0$. These three situations will now be discussed. First case (a), which may apply to the open-packed Bi crystal faces. The long-range atomic interactions now lead to an effective attractive force between the interfaces. The growth of disordered film thickness with increasing temperature is first logarithmic, but then stops beyond the cross-over thickness $l_{c}$. The equilibrium thickness $\bar{l}$ does not diverge as $T_{\mathrm{m}}$ is approached. Instead there is a discontinuous transition at $T=$ $T_{\mathrm{m}}$, resulting in a first-order or incomplete melting (wetting) transition.

Secondly consider case (b), $\Delta \gamma^{\text {SR }}<0$ and $W>$ 0 . The long-range contribution is repulsive whereas the short-range and undercooling terms are attractive. The subtle interplay between the long-range and short-range atomic interactions can give rise to a prewetting transition $[16,43]$ if $\left|\Delta \gamma^{\mathrm{SR}}\right|<$ $\Delta \gamma^{\text {I.R }}$ and $\Delta \gamma^{\mathrm{LR}}>0$, so that $\Delta \gamma^{\mathrm{T}}=\Delta \gamma^{\mathrm{SR}}+\Delta \gamma^{\mathrm{LR}}$ $>0$. The quasi-liquid layer thickness first jumps at a certain temperature to a finite thickness and then diverges as $T_{\mathrm{m}}$ is approached. The layer thickness dependence of the surface free energy $F_{\mathrm{s}}(l)$ is schematically shown in fig. 9. For temperatures well below $T_{\mathrm{m}}$ the undercooling term dominates and increases linearly with increasing $l$ (fig. 9a). As a result, at equilibrium, the surface is well-ordered and the surface free energy is given 


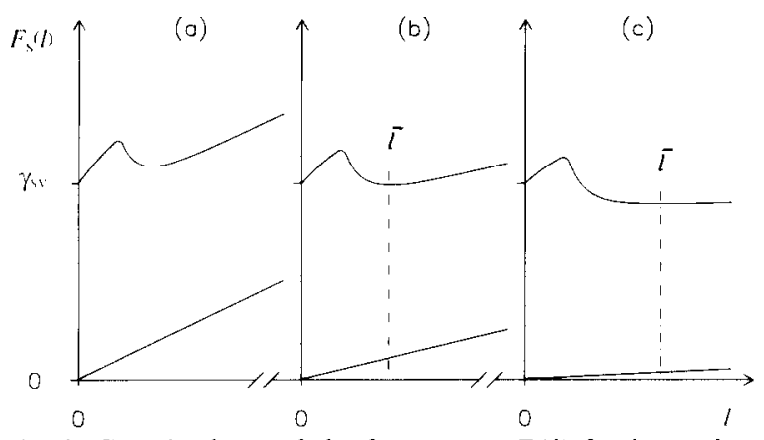

Fig. 9. Generic shape of the free energy $F_{\mathrm{s}}(l)$ for increasing temperatures (a) to (c). The $l$-dependence of $F_{\mathrm{s}}(l)$ is characteristic for a prewetting transition as described in the text.

by $F_{\mathrm{s}}(l=i=0)=\gamma_{\mathrm{sv}}$. Note that $\gamma_{\mathrm{sv}}$ consists of a short and long-range contribution, with $\gamma_{\mathrm{sv}}=\gamma_{\mathrm{sv}}^{\mathrm{SR}}$ $+\gamma_{\mathrm{s} v}^{\mathrm{LR}}$. As the temperature increases, the undercooling term becomes smaller and, at a certain temperature, $F_{\mathrm{s}}(l)$ exhibits two minima of equal depth at zero and nonzero value $l$ (see fig. 9b). A further increase of the temperature results first in a discontinuous change in equilibrium film thickness from $\bar{l}=0$ to $\bar{l}>0$. From then onwards the layer thickens continuously according to a power law and diverges as $T_{\mathrm{m}}$ is approached (fig. 9c). For $\mathrm{Pb}$ such a prewetting transition is possible for crystal face orientations near $\{111\}$ and $\{100\}$ because $\Delta \gamma^{\mathrm{SR}}<0$ for these orientations. But it is difficult to find surface orientations for which $\left|\Delta \gamma^{\mathrm{SR}}\right|<\Delta \gamma^{\mathrm{LR}}$. An obvious choice would be $\mathrm{Pb}\{h k l\}$ surfaces with an orientation deviating by $\sim 8.5^{\circ}$ from $\{111\}$ or by $\sim 5^{\circ}$ from $\{100\}$ because for these crystal faces $\left|\Delta \gamma^{\mathrm{SR}}\right|$ is very small (see Part $\mathrm{I}$ of this investigation). A prewetting transition for $\mathrm{Pb}\{100\}$ has also been predicted by Chernov and Mikheev [44].

Thirdly we discuss situation (c): $\Delta \gamma^{\mathrm{SR}}<0$ and $W<0$, i.e., $\Delta \gamma^{\mathrm{T}}=\Delta \gamma^{\mathrm{SR}}+\Delta \gamma^{\mathrm{LR}}<0$. We expect that this applies to the pure semiconductors such as $\mathrm{Ge}$ and $\mathrm{Si}$, and to the close-packed Bi surfaces. Then both the short-range as the long-range contributions to $F_{\mathrm{s}}(l)$ are against quasi-liquid layer formation and the surface will not melt. We note that recent electron diffraction [45], X-ray diffraction [46] and medium-energy ion scattering expcriments [47] on $\mathrm{Ge}\{111\}$ revealed a loss of crystalline order in the outermost atomic layers at tem- peratures $\sim 150 \mathrm{~K}$ below $T_{\mathrm{m}}$. This result appears to be in conflict with the above prediction. However, the disordered layer thickness was found not to diverge but to level off upon approaching $T_{\mathrm{m}}$ $[45,47]$. The disordering of this surface is probably caused by an atomic-scale structural rearrangement not related to surface-induced melting.

\section{Conclusions}

By expressing the various parameters entering the Landau free energy in terms of known interfacial free energies we have derived clear-cut thermodynamic criteria for the appearance of surface melting and freezing. These criteria indicate a very different behavior of the surface with regard to melting and freezing and account for the general absence of superheating effects in solids. Explicit expressions for the crystal-face and temperature dependence of the melted (frozen) layer thickness are obtained and the calculations are in quantitative agreement with recent observations of melting at differently oriented $\mathrm{Pb}$ surfaces.

\section{Acknowledgements}

We thank R. Lipowsky, J.W.M. Frenken, L.V. Mikheev and A.W. Denier van der Gon for stimulating discussions. This work is part of the research program of the Stichting voor Fundamenteel Onderzoek der Materie (Foundation for Fundamental Research on Matter) and was made possible by financial support from the Nederlandse Organisatie voor Wetenschappelijk Onderzoek (Netherlands Organization for Scientific Research).

\section{Appendix A. Correlation length within the liquid phase}

Within the framework of the Landau theory the correlation length within the liquid phase is given by $\xi_{\ell}=(J / \alpha)^{1 / 2}$. Using eq. (28) we express $\xi_{\ell}$ as $\xi_{\ell}=\frac{4 \gamma_{\mathrm{sl}}}{\alpha M_{\mathrm{cr}}^{2}}$ 
In this appendix we calculate $\xi_{\ell}$ from eq. (A.1) by estimating the values for the parameters $M_{\mathrm{cr}}, \alpha$ and $\gamma_{\mathrm{sl}}$.

First consider $M_{\mathrm{cr}}$ which is the order parameter in the bulk of the crystal lattice. For $M_{\text {cr }}$ we take the Fourier component of the density which corresponds to the smallest nonzero reciprocal lattice vector $G_{1}$ of the crystal. The density of the crystal can be expanded in the Fourier series

$\rho(\boldsymbol{r})=\frac{N}{V} \sum_{j} M_{j} \mathrm{e}^{\mathrm{i} \boldsymbol{G}_{j} \cdot \boldsymbol{r}}$,

with $N$ the number of atoms, $V$ the volume of the crystal, and $\left\{\boldsymbol{G}_{j}\right\}$ the set of reciprocal lattice vectors. The Fourier coefficients

$M_{j}=\frac{1}{N} \int_{V} \rho(\boldsymbol{r}) \mathrm{e}^{-\mathrm{i} G_{j} \cdot \boldsymbol{r}} \mathrm{d}^{3} \boldsymbol{r}$

form a set of order parameters $\left\{M_{j}\right\}$, with $M_{1} \equiv$ $M_{\mathrm{cr}}$. For a lattice of $N$ discrete points, $\rho(\boldsymbol{r})$ is given by

$\rho(\boldsymbol{r})=\sum_{i=1}^{N} \delta\left(\boldsymbol{r}-\boldsymbol{R}_{i}\right)$

with $\boldsymbol{R}_{i}$ a lattice vector given by $\boldsymbol{R}_{i}=x_{i} a+y_{i} b+$ $z_{i} c$, with $x_{i}, y_{i}$ and $z_{i}$ integers and $\boldsymbol{a}, \boldsymbol{b}$ and $\boldsymbol{c}$ the primitive lattice vectors. By substituting eq. (A.4) into eq. (A.3) we obtain

$M_{1} \equiv M_{\mathrm{cr}}=\frac{1}{N} \sum_{i=1}^{N} \mathrm{e}^{-\mathrm{i} G_{1} \cdot R_{i}}=1$.

Secondly we consider the parameter $\alpha$ entering eq. (A.1). Its value can be estimated by using the Hansen-Verlet melting rule. This rule states that the height of the first peak $S\left(Q_{1}\right)$ of the liquid structure factor $S(Q)$ near melting equals $S\left(Q_{1}\right)$ $=2.85$ [48]. The structure factor at $Q=Q_{1}$ is given by [49]

$S\left(\boldsymbol{Q}_{1}\right)=\frac{1}{N}\left\langle\sum_{i=1}^{N}\left|\mathrm{e}^{-\mathrm{i} \boldsymbol{Q}_{1} \cdot \boldsymbol{r}_{i}}\right|^{2}\right\rangle=N\left\langle\left|M_{\ell}\right|^{2}\right\rangle$,

with $r_{i}$ the position of the particle labelled $i$. Here $\left|M_{\ell}\right|$ is the order parameter in the liquid phase at the reciprocal vector $Q_{1}$ for which $S(Q)$ has its maximum. The length of $\boldsymbol{Q}_{1}$ is given by $\left|\boldsymbol{Q}_{1}\right|=$ $2 \pi / \Delta r \approx\left|G_{1}\right|$, with $\Delta r$ being the location of the first peak in the pair distribution function [49].
Note that the definition of $\left|M_{\ell}\right|$, as given by eq. (A.6), is consistent with the definition of the order parameter $M_{\mathrm{cr}}$ in the crystal lattice. That is, in the limit of perfect crystalline order, $\left|M_{\ell}\right| \rightarrow 1$ (eq. (A.5)). The ensemble-average $\left\langle\left|M_{\ell}\right|^{2}\right\rangle$ is given by

$$
\begin{aligned}
\left\langle\left|M_{\ell}\right|^{2}\right\rangle= & \int_{0}^{2 \pi} \mathrm{d} \varphi \int_{0}^{+\infty}\left|M_{\ell}\right| \mathrm{d}\left|M_{\ell}\right| P\left(\left|M_{\ell}\right|\right) \\
& \times\left|M_{\ell}\right|^{2},
\end{aligned}
$$

with $P\left(\left|M_{\ell}\right|\right)$ the probability to find the liquid in the state with order parameter $\left|M_{\ell}\right|$. At $T=T_{\mathrm{m}}$, $P\left(\left|M_{\ell}\right|\right)$ is given by the Boltzmann distribution

$P\left(\left|M_{\ell}\right|\right)=A \exp \left(-\frac{f\left(\left|M_{\ell}\right|\right) V}{k_{\mathrm{B}} T_{\mathrm{m}}}\right)$.

The parameter $A$ is a constant which follows from the normalization condition

$$
\int_{0}^{2 \pi} \mathrm{d} \varphi \int_{0}^{+\infty}\left|M_{\ell}\right| \mathrm{d}\left|M_{\ell}\right| P\left(\left|M_{\ell}\right|\right)=1
$$

We now assume that it is $\left|M_{\ell}\right|$ which enters eq. (2a) for the Landau free energy. Inserting the Landau expression $f\left(\left|M_{\ell}\right|\right)=\alpha\left|M_{\ell}\right|^{2} / 2$ (eq. (2a) with $\Lambda=0$ ) in eq. (A.8), we obtain for $P\left(\left|M_{\ell}\right|\right)$ the normalized distribution

$$
P\left(\left|M_{\ell}\right|\right)=\frac{\alpha V}{2 \pi k_{\mathrm{B}} T_{\mathrm{m}}} \exp \left(\frac{-\alpha\left|M_{\ell}\right|^{2} V}{2 k_{\mathrm{B}} T_{\mathrm{m}}}\right) .
$$

Substitution of eq. (A.10) into (A.7) gives

$\left\langle\left|M_{\ell}\right|^{2}\right\rangle=2 k_{\mathrm{B}} T_{\mathrm{m}} / \alpha V$.

The expression for the structure factor at $\boldsymbol{Q}=\boldsymbol{Q}_{1}$ is obtained by substitution of eq. (A.11) into eq. (A.6)

$S\left(Q_{1}\right)=2 \rho_{\ell} k_{\mathrm{B}} T_{\mathrm{m}} / \alpha$,

where $\rho_{\ell}=N / V$ is the average particle number density in the liquid phase. By using the HansenVerlet melting rule, $S\left(Q_{1}\right)=2.85$, we obtain from eq. (A.12)

$\alpha=2 \rho_{\ell} k_{\mathrm{B}} T_{\mathrm{m}} / 2.85$.

The correlation length $\xi_{\ell}$ within the liquid phase can now be estimated. By substituting $M_{\mathrm{cr}}=1$ (eq. 
(A.5)) and the expression for $\alpha$ (eq. (A.13)) into eq. (A.1) we obtain

$\xi_{\ell}=5.7 \gamma_{\mathrm{s}} / \rho_{\ell} k_{\mathrm{B}} T_{\mathrm{m}}$.

This equation expresses $\xi_{\ell}$ in terms of known thermodynamic quantities. For $\mathrm{Pb}, \gamma_{\mathrm{st}}$ is given by the value $0.04 \pm 0.02 \mathrm{~J} / \mathrm{m}^{2}$, which is the average over different experimental values $[18,20,21]$. Taking $\rho_{\ell}=3.11 \times 10^{28} \mathrm{~m}^{-3}$ and $T_{\mathrm{m}}=600.7 \mathrm{~K}$ we calculate for $\xi_{\ell}$ the value $\xi_{\ell}=0.9 \pm 0.4 \mathrm{~nm}$. This value is, to within the error of measurement, in agreement with the value of $\xi_{\ell}=0.63 \mathrm{~nm} \mathrm{[6]} \mathrm{mea-}$ sured on $\mathrm{Pb}\{110\}$ and with the values of $\xi_{\ell}=0.47$ $\pm 0.09 \mathrm{~nm}$ and $\xi_{\ell}=0.61 \pm 0.09 \mathrm{~nm}$ which represent averages over different $\mathrm{Pb}$ crystal planes lying in the $\langle 110\rangle$ and $\langle 100\rangle$ crystallographic zones, respectively (see part I of this investigation). We note that, according to eq. (A.14), the value for $\xi_{\ell}$ is crystal-face dependent through the anisotropic solid-liquid interface free energy $\gamma_{s}\{h k l\}$. Such a trend has indeed been observed in the medium-energy ion scattering measurements reported in part I of this investigation (ref. [40] therein).

\section{Appendix B. Effect of thermal fluctuations on the melt thickness}

The mean-field theory presented here does not account for the possible effect of thermal fluctuations. The surface free energy $F_{\mathrm{s}}(l)$, given by eq. (34), describes the interaction between two interfaces which are assumed to be planar. However, the solid-liquid interfaces of many materials, especially of metals, are predicted to be rough [50] and the presence of thermally excited capillary waves on free liquid surfaces is well-established [51,52]. Thermally induced fluctuations in semi-infinite systems have been studicd theoretically [5358]. Below we give an estimate for the magnitude of the thermally induced interface roughness for a system governed by short-range atomic interactions. We also estimate the amount by which the mean-field result for the quasi-liquid layer thickness changes if fluctuations are taken into account.

Consider a surface melting transition in a system with a rough solid-quasi-liquid interface. For small melt thickness the thermal fluctuations of this interface are damped by the presence of the quasi-liquid-vapor interface [59]. As the melt thickness increases the fluctuations become less constraint. This leads to a reduction in free energy, which can be associated with a fluctuationinduced repulsive interaction between the two respective interfaces. It has been shown that this repulsion is short-ranged; it falls off as $\exp \left(\cdots 4 \pi \zeta_{\perp}^{2} \Sigma / k_{\mathrm{B}} T\right)$, with $\zeta_{\perp}$ rcpresenting the roughness perpendicular to the average quasiliquid-solid interface $[54,55]$. The parameter $\Sigma$ represents the stiffness of the solid-liquid interface, with $\gamma_{\mathrm{sl}}<\Sigma<\infty[55,58]$. Whether or not the thermal fluctuations can be ignored depends on the importance of the fluctuation-induced interaction relative to the repulsive interaction term in $F_{\mathrm{s}}(l)$, which falls off with distance $l$ as $\exp (-2 \kappa l)$ (see eq. (34)). The fluctuation-induced repulsion should be taken into account if $4 \pi \zeta_{\perp}^{2} \Sigma / k_{\mathrm{B}} T$ is no longer large with respect to $2 \kappa l$. The equilibrium melt thickness $\bar{l}$ then changes to $[3,53,60]$

$\bar{l}^{*} \approx\left(1+\tau^{2} / 2 \pi\right) \bar{l}$,

with $i$ the mean-field thickness (eq. (33)) and $i^{*}$ the corrected thickness corresponding to the average equilibrium position of the rough quasiliquid-solid interface. The interfacial roughness $\zeta_{\perp}[3]$ is given by

$\zeta_{\perp}^{2} \approx\left(\tau^{2} \xi_{\ell} / 2 \pi\right) \bar{l}$

and the dimensionless parameter $\tau$ is given by

$\tau^{2} \approx k_{\mathrm{B}} T_{\mathrm{m}} / \Sigma \xi_{\ell}^{2}$,

provided $\tau<(2 \pi)^{1 / 2}$. For a smooth interface the stiffness $\Sigma$ is infinite so that $\tau \rightarrow 0$ and the meanfield results are recovered. For finite $\Sigma$ the interface is rough. We note that the thermal fluctuations only modify the factor in front of the logarithmic growth law (eqs. (33) and (B.1)), not the growth law itself.

Let us now consider the $\mathrm{Pb}\{110\}$ surface. We take for $\Sigma$ the minimum value $\Sigma=\gamma_{\mathrm{sl}} \approx 0.05 \mathrm{~J} / \mathrm{m}^{2}$ and for the correlation length the value $\xi_{\ell}=0.63$ $\mathrm{nm}$ [6]. From eq. (B.3) we obtain for $\tau$ a value of 0.6 , which is indeed smaller than $(2 \pi)^{1 / 2}$. By substituting this valuc of $\tau$ into cq. (B.1) we find that the maximum correction to the melted layer thickness $\bar{l}$ is only 7\%. For a typical film thickness of 
$\bar{l}=2 \mathrm{~nm}$ we obtain from eq. (B.2) an interfacial roughness of $\zeta_{\perp} \approx 0.3 \mathrm{~nm}$. This value is small and comparable to the atomic diameter of $\mathrm{Pb}(0.35$ $\mathrm{nm}$ ). For most other elements listed in table 1 the corrections to the mean-field results are expected to be even smaller than for $\mathrm{Pb}$. This is caused by the larger solid-liquid interfacial free energies for the other elements, see table 1.

\section{Glossary of symbols}

$\begin{array}{ll}T & \begin{array}{l}\text { temperature } \\ \text { triple point }\end{array} \\ T_{\mathrm{m}} & \begin{array}{l}\text { solid-vapor interfacial free energy per } \\ \text { unit area } \\ \gamma_{\mathrm{sv}}\end{array} \\ \gamma_{\mathrm{sl}} & \begin{array}{l}\text { solid-liquid interfacial free energy per } \\ \text { unit area }\end{array} \\ \gamma_{\mathrm{lv}} & \begin{array}{l}\text { liquid-vapor interfacial free energy per } \\ \text { unit area }\end{array} \\ \Delta \gamma & \begin{array}{l}\text { free energy per unit area which the } \\ \text { ordered solid surface has in excess of a } \\ \text { surface completely wetted by an in- } \\ \text { finitely thick liquid layer }\left(\Delta \gamma=\gamma_{\mathrm{sv}}-\gamma_{\mathrm{sl}}\right.\end{array} \\ & \left.-\gamma_{\mathrm{lv}}\right)\end{array}$

$\Delta \gamma^{\prime} \quad$ free energy per unit area which the liquid surface has in excess of a liquid surface covered by a infinitely thick solid layer $\left(\Delta \gamma^{\prime}=\gamma_{\mathrm{lv}}-\gamma_{\mathrm{sl}}-\gamma_{\mathrm{sv}}\right)$

$M(z) \quad$ order parameter profile

$M_{\mathrm{S}} \quad$ order parameter $M(z=0)$ at the surface

$M_{\text {cr }} \quad$ order parameter of the bulk in its crystalline state

$f(M) \quad$ free energy per unit volume for homogeneous bulk material as a function of order parameter

$f_{1}\left(M_{\mathrm{S}}\right) \quad$ free energy per unit surface area as a function of the surface order parameter

\section{$L_{\mathrm{m}}$}

$\Lambda$ latent heat of melting per unit volume $=L_{\mathrm{m}}\left(1-T / T_{\mathrm{m}}\right) ; \Lambda$ is the difference in free energy per unit volume between solid and liquid at temperature $T$

$\xi_{\ell} \quad$ correlation length within the liquid phase $\left(\xi_{\ell}=\kappa^{-1}\right)$

$l \quad$ thickness of quasi-liquid layer

$i$ equilibrium thickness of quasi-liquid layer

$s \quad$ thickness of quasi-solid layer $\bar{s}$

$W$

$\left\{\boldsymbol{G}_{j}\right\}$

$\rho(\boldsymbol{r})$

$\rho_{\ell}$

$S(Q)$ equilibrium thickness of quasi-solid layer

Hamaker constant

set of reciprocal lattice vectors

local particle density

average particle density in the liquid structure factor of the liquid

\section{References}

[1] J.F. van der Veen, B. Pluis and A.W. Denier van der Gon, in: Chemistry and Physics of Solid Surfaces VII, Vol. 10 of Springer Series in Surface Sciences, Eds. R. Vanselow and R.F. Howe (Springer, Heidelberg, 1988) p. 455, and references therein.

[2] R. Lipowsky and W. Speth, Phys. Rev. B 28 (1983) 3983; R. Lipowsky, Phys. Rev. Lett. 49 (1982) 1575.

[3] R. Lipowsky, Ferroelectrics 73 (1987) 69, and references therein.

[4] J.N. Israelachvili, Intermolecular and Surface Forces (Academic, San Diego, 1985).

[5] B. Pluis, A.W. Denier van der Gon, J.W.M. Frenken and J.F. van der Veen, Phys. Rev. Lett. 59 (1987) 2678.

[6] B. Pluis, T.N. Taylor, D. Frenkel and J.F. van der Veen, Phys. Rev. B 40 (1989) 1353.

[7] B. Pluis, A.W. Denier van der Gon, J.F. van der Veen and A.J. Riemersma, Surf. Sci. 239 (1990) 265.

[8] J.W.M. Frenken and J.F. van der Veen, Phys. Rev. Lett. 54 (1985) 134.

[9] J.W.M. Frenken, P.M.J. Marée and J.F. van der Veen, Phys. Rev. B 34 (1986) 7506.

[10] Da-Ming Zhu and J.G. Dash, Phys. Rev. Lett. 57 (1986) 2959.

[11] Da-Ming Zhu and J.G. Dash, Phys. Rev. Lett. 60 (1988) 432.

[12] A.W. Denier van der Gon, R.J. Smith, J.M. Gay, D.J. O'Connor and J.F. van der Veen, Surf. Sci. 227 (1990) 143.

[13] J.W. Cahn and J.E. Hilliard, J. Chem. Phys. 28 (1958) 258.

[14] T. Meister and H. Müller-Krumbhaar, Phys. Rev. Lett. 51 (1983) 1780.

[15] See for example: H. Margenau and G.M. Murphy, The Mathematics of Physics and Chemistry (Van Nostrand, New York, 1951) p. 195.

[16] S. Dietrich, in: Phase Transitions and Critical Phenomena, Vol. 12, Eds. C. Domb and J.L. Lebowitz (Academic Press, London, 1988), and references therein.

[17] Usually, "drying" corresponds to the intrusion at coexistence of a layer of gas between the (bulk) liquid and the solid substrate [R. Pandit, M. Schick and M. Wortis, Phys. Rev. B 26 (1982) 5112]. However, in the context of surface freezing we define drying as the intrusion at the triple point of a layer of solid between bulk gas and liquid substrate. 
[18] D.P. Woodruff, The Solid-Liquid Interface (Cambridge University Press, London, 1973), and references therein.

[19] D.R.H. Jones, J. Mater. Sci. 9 (1974) 1, and references therein.

[20] D. Turnbull, J. Appl. Phys. 21 (1950) 1022

[21] A.R. Miedema and F.J.A. den Broeder, Z. Metallk. 70 (1979) 14, and references therein.

[22] A.R. Miedema and R. Boom, Z. Metallk. 69 (1978) 183, and references therein.

[23] A.R. Miedema, Z. Metallk. 69 (1978) 287, and references therein.

[24] Handbook of Chemistry and Physics, 62th ed., Eds. R.C. Weast and M.J. Astle (CRC Press, Boca Raton, 1981).

[25] K.C. Prince, U. Breuer and H.P. Bonzel, Phys. Rev. Lett. 60 (1988) 1146.

[26] U. Breuer, H.P. Bonzel, K.C. Prince and R. Lipowsky, Surf. Sci. 223 (1989) 258.

[27] P.H. Fuoss, L.J. Norton and S. Brennan, Phys. Rev. Lett. 60 (1988) 2046.

[28] G.D.T. Spiller, Philos. Mag. A 46 (1982) 535.

[29] K.D. Stock, Surf. Sci. 91 (1980) 655;

K.D. Stock and E. Menzel, Surf. Sci. 61 (1976) 272.

[30] P. von Blanckenhagen, W. Schommers and V. Voegele, J. Vac. Sci. Technol. A 5 (1987) 649.

[31] G. Fritsch, R. Lachner, H. Diletti and E. Lüscher, Philos. Mag. A 46 (1982) 829.

[32] J.C. Heyraud and J.J. Métois, Surf. Sci. 128 (1983) 334.

[33] J.C. Heyraud and J.J. Métois, Surf. Sci. 177 (1986) 213.

[34] J.C. Heyraud and J.J. Métois, Acta Met. 28 (1980) 1789.

[35] M. McLean and H. Mykura, Surf. Sci. 5 (1966) 466.

[36] T. Barsotti, J.M. Bermond and M. Drechsler, Surf. Sci. 146 (1984) 467.

[37] B. Pluis, J.M. Gay, J.W.M. Frenken, S. Gierlotka, J.F. van der Veen, J.E. Macdonald, A.A. Williams, N. Piggins and J. Als-Nielsen, Surf. Sci. 222 (1989) L845.

[38] B.Q. Lu, R. Evans and M.M. Telo da Gama, Mol. Phys. 55 (1985) 1319.

[39] J.A. Barker and J.R. Henderson, J. Chem. Phys. 76 (1982) 6303.
[40] J.A. Støvneng, T. Aukrust and E.H. Hauge, Physica A 143 (1987) 40.

[41] D.E. Sullivan and M.M. Telo da Gama, in: Fluid Interfacial Phenomena, Ed. C.A. Croxton (Wiley, New York, 1986).

[42] A.A. Chernov and V.A. Yakovlev, Langmuir 3 (1987) 635.

[43] H. Nakanishi and M.E. Fisher, Phys. Rev. Lett. 49 (1982) 1565.

[44] A.A. Chernov and L.V. Mikheev, Physica A 157 (1989) 1042.

[45] E.G. McRae and R.A. Malic, Mhys. Rev. Lett. 58 (1987) 1437.

[46] R.G. van Silfhout et al., unpublished.

[47] A.W. Denier van der Gon, J.M. Gay, J.W.M. Frenken and J.F. van der Veen, to be published.

[48] J.P. Hansen and D. Schiff, Mol. Phys. 25 (1973) 1281.

[49] J.P. Hansen and I.R. McDonald, Theory of Simple Liquids (Academic Press, London, 1986).

[50] K.A. Jackson, in: Progress in Solid State Chemistry, Vol. 4, Ed. H. Reiss (Pergamon, New York, 1967).

[51] D. Beaglehole, Phys. Rev. Lett. 58 (1987) 1434, and references therein.

[52] A. Braslau, M. Deutsch, P.S. Pershan, A.H. Weiss, J. Als-Nielsen and J. Bohr, Phys. Rev. Lett. 54 (1985) 114.

[53] D.S. Fisher and D.A. Huse, Phys. Rev. B 32 (1985) 247, and references therein.

[54] R. Lipowsky and M.E. Fisher, Phys. Rev. B 36 (1987) 2126, and references therein.

[55] R. Lipowsky, Habilitations-Schrift, Ludwig-MaximiliansUniversität, München, 1987 (distributed by KFA Jülich as Jül-Spez-438), and references therein.

[56] R. Lipowsky, Phys. Rev. Lett. 52 (1984) 1429.

[57] A.A. Chernov and L.V. Mikheev, Phys. Rev. Lett. 60 (1988) 2488.

[58] M.E. Fisher and D.S. Fisher, Phys. Rev. B 25 (1982) 3192.

[59] A.C. Levi and E. Tosatti, Surf. Sci. 178 (1986) 425.

[60] E. Brézin, B.I. Halperin and S. Leibler, Phys. Rev. Lett. 50 (1983) 1387. 